\title{
Assessment of the performance of the inter-arrival time algorithm to identify ice shattering artifacts in cloud particle probe measurements
}

\author{
A. Korolev ${ }^{1}$ and P. R. Field ${ }^{2}$ \\ ${ }^{1}$ Environment Canada, Toronto, Canada \\ ${ }^{2}$ Met Office, Exeter, UK, and University of Leeds, Leeds, UK \\ Correspondence to: A. Korolev (alexei.korolev@ec.gc.ca)
}

Received: 18 August 2014 - Published in Atmos. Meas. Tech. Discuss.: 8 October 2014

Revised: 22 December 2014 - Accepted: 20 January 2015 - Published: 17 February 2015

\begin{abstract}
Shattering presents a serious obstacle to current airborne in situ methods of characterizing the microphysical properties of ice clouds. Small shattered fragments result from the impact of natural ice crystals with the forward parts of aircraft-mounted measurement probes. The presence of these shattered fragments may result in a significant overestimation of the measured concentration of small ice crystals, contaminating the measurement of the ice particle size distribution (PSD). One method of identifying shattered particles is to use an inter-arrival time algorithm. This method is based on the assumption that shattered fragments form spatial clusters that have short inter-arrival times between particles, relative to natural particles, when they pass through the sample volume of the probe. The inter-arrival time algorithm is a successful technique for the classification of shattering artifacts and natural particles. This study assesses the limitations and efficiency of the inter-arrival time algorithm. The analysis has been performed using simultaneous measurements of two-dimensional (2-D) optical array probes with the standard and antishattering "K-tips" collected during the Airborne Icing Instrumentation Experiment (AIIE). It is shown that the efficiency of the algorithm depends on ice particle size, concentration and habit. Additional numerical simulations indicate that the effectiveness of the inter-arrival time algorithm to eliminate shattering artifacts can be significantly restricted in some cases. Improvements to the inter-arrival time algorithm are discussed. It is demonstrated that blind application of the inter-arrival time algorithm cannot filter out all shattered aggregates. To mitigate against the effects of shattering, the inter-arrival time algorithm should be used together
\end{abstract}

with other means, such as antishattering tips and specially designed algorithms for segregation of shattered artifacts and natural particles.

\section{Introduction}

Ice particle size distributions (PSDs) are used in atmospheric models for computing both the radiative impact of ice clouds and the microphysical process rates that control ice cloud evolution. Therefore, an inaccurate representation of the ice PSD can have adverse impacts on the accuracy of climate and weather prediction models. Measurements of ice PSDs from aircraft-based observations form the basis of size distribution representations in models. Prior to entering the instrument sample volume, an ice particle may impact the probe's upstream tips or inlet and shatter into many small fragments. These shattering products can then significantly contaminate measurements of airborne particle probes.

Over three decades the abundance of small ice particles measured by particle probes in the tropospheric clouds remained an intriguing problem. Recent studies using simultaneous measurements of standard and modified probes (Korolev et al., 2011, 2013b) have unambiguously demonstrated that in many cases measurements made with standard cloud probes are adversely affected by shattering artifacts.

There are two main approaches currently used within the cloud physics community to mitigate the effect of shattering. One approach is based on modifying the inlet configuration of the cloud probes to minimize the area that deflects shat- 
tered particles towards the sample volume (Korolev et al., 2013a). This method can lead to a significant reduction in the effect of shattering but is not able to completely eradicate the problem (Korolev et al., 2011, 2013b; Lawson, 2011).

The second approach is a postprocessing methodology based on the fact that shattering products are spatially clustered. Because of close spacing, the time difference between two successive shattered fragments passing through the sample volume will be much shorter than that for naturally occurring particles. This time difference is usually referred to as "inter-arrival time".

Cooper (1977) suggested that these artifacts could be filtered out by identifying the characteristically short interarrival times of particles associated with these shattering products.

Field et al. (2003) used a fast forward scattering spectrometer probe (FSSP) to measure particle spacing in ice clouds. The inter-arrival time distribution in ice clouds was found to have a bimodal shape with modes at $10^{-2}$ and $10^{-4} \mathrm{~s}$ corresponding to approximately $1 \mathrm{~m}$ and $1 \mathrm{~cm}$ spatial separations. The particles from the long and short inter-arrival time modes corresponded to estimated concentrations of $0.1-1 \mathrm{~cm}^{-3}$ and $\sim 100 \mathrm{~cm}^{-3}$ respectively. No conclusions were drawn as to whether the latter localized clusters of high particle concentration were natural or artifacts. Assuming they were artifacts, their inter-arrival time algorithm suggested average and maximum concentration overestimates of a factor of 2 and 5 respectively.

Field et al. (2006) applied an inter-arrival time algorithm (ITA) to filter out shattering artifacts, choosing a threshold inter-arrival time in the range $10^{-4}$ to $10^{-5} \mathrm{~s}$, depending on the instrument and the aircraft type used for the data collection, to reject the short inter-arrival mode. It was found that the OAP-2DC and CIP concentrations were reduced by up to a factor of 4 when the mass-weighted mean size exceeded $3 \mathrm{~mm}$. The ice water content (IWC) estimate was reduced by up to $20-30 \%$, most notable in cases of narrow size distributions. It was also found that the corrected PSDs could show a reduction in particle concentrations over a wide range of sizes from 200 microns for narrow distributions up to 1000 microns for the broadest distributions that are subject to the most shattering.

It should be noted that the time separation between particle arrivals through the probe's sample volume depends on true air speed. The relevant metric, which should be used to segregate shattered artifacts and natural particles is interparticle distance along the flight direction $(\Delta x)$. It is acknowledged that the community is accustomed to referring to "inter-arrival time" $\Delta t$ since this parameter is measured in the probes. In the following discussion we will be using both metrics $\Delta x$ and $\Delta t$.

The ITA is now routinely used in two-dimensional (2-D) probe data processing to eliminate shattering artifacts (e.g., Baker et al., 2009; Lawson, 2011; Jackson et al., 2014). Moreover, it is recognized that the best practice for the op- eration of cloud probes in the presence of ice is provided by a combination of modified tips and the application of the inter-arrival time filtering together (e.g., Korolev et al., 2011; Baumgardner et al., 2012).

While it has been demonstrated that the ITA reduces the effects of shattering, its accuracy and efficiency remains poorly quantified. For instance, can the ITA alone identify all shattering artifacts? Is the efficiency of the ITA dependent upon the probe specifications such as pixel resolution, response time, sample area, and inlet configuration?

Motivation for improving our understanding of the limitations and efficiency of the ITA is threefold. Firstly, there is a need to determine if the ITA can be used to successfully reanalyze the historical data collected over the past thirty years. Secondly, an improved quantitative understanding of the limitations of the ITA will provide statements of the accuracy of the measurements of the particle number concentration, ice water content, extinction coefficient and other PSD derived parameters. Thirdly, knowledge of the efficiency of the ITA will aid in the design process of future cloud probes.

The objective of this paper is to evaluate the efficiency and limitations of ITA. A detailed description and the main assumptions underlying the algorithm are presented in Sect. 2. Section 3 considers general limitations of the algorithm. Analysis of the results of the OAP-2DC data processing using the ITA are presented in Sect. 4. In Sect. 5 statistical simulations of ice particle shattering are used to explore the limitations of the ITA. Finally, Sect. 6 provides a summary.

\section{Description of the inter-arrival time algorithm}

\subsection{Basic assumptions}

In the following discussion the term "shattering event" will be applied to the group of shattered fragments, that (a) formed as a result of impact of a single particle with the upstream tips (or inlet) of a probe, and (b) at least one particle from the group of the shattered fragments was registered by the probe. It should be noted that if the particle rebounds to the sample area without shattering, it still falls in the definition of the "shattering event".

The successful application of the ITA is predicated on two basic assumptions: (1) the maximum inter-arrival time of the shattered fragments is shorter than the minimum inter-arrival time between intact particles; (2) shattered particles are always passing through the sample volume as a group of no less than two particles.

The first assumption is a necessary condition for the complete separation of the inter-arrival time distributions $\phi_{\mathrm{S}}(\Delta t)$ and $\phi_{\mathrm{i}}(\Delta t)$ without overlap. Here $\phi_{\mathrm{s}}(\Delta t)$ and $\phi_{\mathrm{i}}(\Delta t)$ are the distribution of inter-arrival times associated with shattering events and intact particles, respectively. The absence of an overlap between $\phi_{\mathrm{S}}(\Delta t)$ and $\phi_{\mathrm{i}}(\Delta t)$ allows for the existence of a cut-off time $\tau^{*}$ such that all shattered and only shattered 

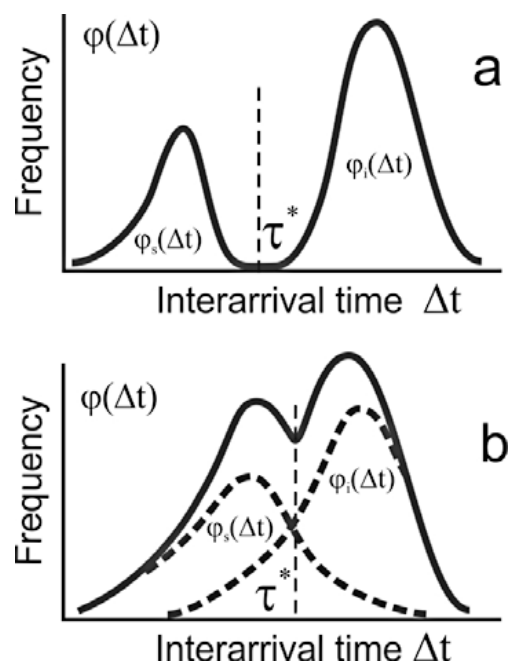

Figure 1. Conceptual diagram of the distribution of inter-arrival times $\phi(\Delta t)$ with well separated short and long inter-arrival time modes (a), when shattering artifacts can be segregated from the intact particles. When the distributions of inter-arrival time associated with intact particles $\phi_{\mathrm{i}}(\Delta t)$ and shattered fragments $\phi_{\mathrm{S}}(\Delta t)$ have significant overlap, then segregation of intact particles and shattered artifacts is hindered (b).

particles satisfy the condition $\Delta t<\tau^{*}$, whereas all intact particles and only intact particles are associated with $\Delta t>\tau^{*}$ (Fig. 1a). Given this assumption it is trivial to identify the shattered artifacts and intact particles based simply on a comparison of measured inter-arrival time $\Delta t$ between two successive particles and the cut-off time $\tau^{*}$.

The second assumption forms the second necessary condition to identify shattered artifacts. A minimum of two closely spaced particles is necessary to allow artifacts to be identified.

The conceptual diagram in Fig. 2a demonstrates these two basic assumptions about particle spacing that is required for the successful segregation of shattered artifacts and intact particles using an ITA. In reality, the challenges of image sampling and the statistical nature of particle spacings impose limitations on the performance of the ITA in the segregation of shattered artifacts and intact particles. As will be shown below, the first assumption cannot be satisfied due to statistical limitations, whereas the second condition is necessary but not sufficient.

\subsection{Inter-arrival time algorithm}

Here we describe the sequence of operations composing the basic ITA. This algorithm in its basic form will be used in the present study.

1. The inter-arrival time algorithm starts from the calculations of the distribution of inter-arrival times $\phi(\Delta t)$ as in Fig. 1. The calculation of $\phi(\Delta t)$ are performed for each averaging time interval. Similar to Field et
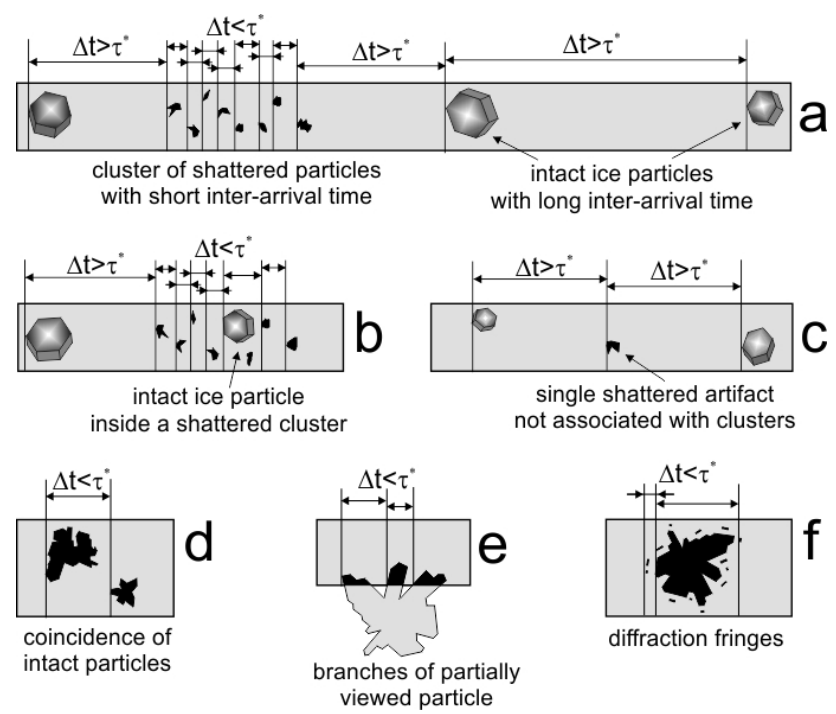

diffraction fringes viewed particle

Figure 2. Conceptual diagram of (a) idealised spatial sequence of intact particles and shattered artifacts passing through the sample volume. Case (c) when the inter-arrival time algorithm may confuse shattered artifact with intact particles, and (b, d, e, f) when intact particles may be confused with shattering artifacts.

al. $(2003,2006)$ the time bins in $\phi(\Delta t)$ were logarithmically spaced. The width of the time bins was optimized to trade-off accuracy of the estimation of $\tau^{*}$ and the statistical significance related to the number of counts in each time bin.

2. The cut-off-time $\tau^{*}$ was calculated for each averaging time interval as a minimum between two maxima associated with long and short time modes (Fig. 1). In cases when only one mode was present, $\tau^{*}$ was forced to be equal to the minimum inter-arrival time found in this averaging interval. It should be noted that the function $\phi(\Delta t)$ is a non-normalized distribution of counts in each time bin. Normalization using the bin width leads to a disappearance of the minimum between the short and long time modes in $\phi(\Delta t)$, which hinders calculation of $\tau^{*}$.

3. Pairs of particles that satisfied the condition $\Delta t<\tau^{*}$ were identified and marked as artifacts. It is important to note that the ITA cannot identify a single-particle shattered artifact (singleton) and that the minimum number of particles identified as artifacts is two.

The value of $\tau^{*}$ should be calculated for each averaging time interval. As will be shown below, $\tau^{*}$ has a wide dynamic range and it depends on many microphysical, environmental and instrumental parameters (Sect. 4.3). The assumption, that $\tau^{*}$ remains constant, may result in large errors in identifying shattering artifacts.

The values of $\tau^{*}$ and $\Delta t$ depend on the airplane speed. In the described algorithm it is assumed that the aircraft speed 
remained approximately constant at each averaging time interval. This assumption works well for a few seconds averaging intervals. However, the aircraft speed depending on the altitude may change by as much as a factor of two during the flight operation. This gives another reason to recalculate $\tau^{*}$ at each averaging time interval.

It is relevant to mention here that alternative techniques for determining $\tau^{*}$ were used by Field et al. (2003, 2006), Lawson (2011) and Jackson et al. (2014). These techniques were based on fitting the function $\phi_{\mathrm{i}}(\Delta t)$ by the Poisson distribution.

\section{Limitations of the inter-arrival time algorithm}

This section presents a list of sampling effects that demonstrate how the assumptions underlying the inter-arrival time algorithm can be contravened. These cases impose limitations on the ability of the ITA to segregate intact particles and shattering artifacts.

In the following we assume that particles are distributed randomly in space and that their spacing and hence interarrival time is well represented by a Poisson distribution. For the Poisson process the density function for counting one intact particle during time $\Delta t$ is described by

$$
\frac{\mathrm{d} P(\Delta t)}{\mathrm{d} t}=\frac{e^{-\Delta t / \tau}}{\tau}
$$

where $\tau=1 / n S u$ is the average inter-arrival time between intact particles passing through the probe's sample area $S ; n$ is the particle concentration; $u$ is the sampling speed. Shattered particles detected by the probe were deflected into the sample area after the impact with the inlet. Therefore, the shattered particles have external origin, are intermittent and their distribution can be considered as independent with respect to the intact particles. Examination of the short interarrival time mode does indicate that these particles also appear to be characterized quite well with a Poisson distribution (e.g., Field et al., 2003, 2006; Sect. 4.3 in this paper).

\subsection{Naturally occurring particles with inter-arrival times shorter than the cut-off-time interval}

Two closely spaced intact particles will be identified as a shattering artifact if the inter-arrival time $\Delta t<\tau^{*}$ (Fig. 2d). Such cases break the first assumption in Sect. 2.1. The probability for coincidence of three or more particles falls very rapidly and has been ignored. The probability of two particles arriving within $\tau^{*}$ can be found from the Poisson statistics as

$P_{2}\left(\Delta t<\tau^{*}\right)=\frac{1}{2}\left(\frac{\tau^{*}}{\tau}\right)^{2} e^{-\frac{\tau^{*}}{\tau}}$

As follows from Eq. (1), the probability of such an event increases with increasing particle concentration $n$ (decreasing $\tau$ ) and increases in the cut-off time $\tau^{*}$.

\subsection{Coincidence of a shattering event particle and an intact particle}

The probability of coincidence of the arrival time of an intact particle during the shattered event (Fig. 2b) can be estimated as

$P\left(\Delta t<\Delta t_{\mathrm{sh}}\right)=\int_{0}^{\Delta t_{\mathrm{sh}}} \frac{e^{-\Delta t / \tau}}{\tau} d(\Delta t)=1-e^{-\Delta t_{\mathrm{sh}} / \tau}$.

Here $\Delta t_{\mathrm{sh}}=\sum_{i} \Delta t_{\mathrm{sh}}(i)=L_{\mathrm{sh}} / u$ is the duration of the shattering event registered by the probe; $\Delta t_{\mathrm{sh}}(i)$ is the interarrival time between two subsequent shattered fragments registered by the probe within the shattered event; $L_{\mathrm{sh}}$ is the spatial length of the shattering event along the flight direction (i.e., distance between the first and last fragments in the shattering event). Equation (2) indicates that even when $\Delta t_{\text {sh }} \ll \tau$, the probability of the arrival of the intact particle in the sample volume during the shattering event remains non-zero. Basically, it means that in principle it is impossible to separate all shattering artifacts and intact particles, and the functions $\phi_{\mathrm{S}}(\Delta t)$ and $\phi_{\mathrm{i}}(\Delta t)$ always overlap (Fig. 1b). The relative fraction of the overlapping area of $\phi_{\mathrm{s}}(\Delta t)$ and $\phi_{\mathrm{i}}(\Delta t)$ characterizes the frequency of misidentifying intact particles and shattering artifacts.

It is possible to attempt to correct for the removal of intact particles using Poisson statistics to estimate the fraction of intact particles rejected and then scale the remaining intact size distribution (e.g., Field et al., 2006).

\subsection{Singletons: single particle shattering artifacts}

A significant limitation of the ITA is related to situations when only one particle from the group of the shattered fragments is registered by the probe (Fig. 2c). Such a situation may occur when most of the shattered fragments travel outside of the sample volume, but a single fragment passes through the sample volume. It may also happen when most of the shattered fragments are smaller than the probe's detecting threshold, and only one particle exceeds the threshold and is registered by the probe. Ice particles may also rebound from the inlet without fragmentation, thus forming a single particle shattering event. Rebounding without shattering was demonstrated in Korolev et al. (2013a, Fig. 13a-d). With respect to the ITA, the single particle artifacts described above can have long inter-arrival times and are therefore indistinguishable from the natural population of intact particles. Due to the random nature of particle impact with the probe's arms and the direction of the trajectories of the rebound shattered fragments the probability of the single particle shattering events always remains non-zero. This imposes a significant and difficult-to-quantify limitation on the performance of the ITA. 

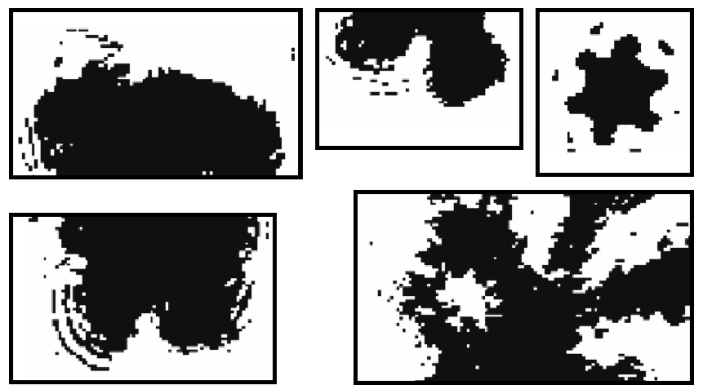

Figure 3. Examples of diffraction fringes around out-of-focus images measured by CIP at $15 \mu \mathrm{m}$ pixel resolution. The diffraction fringes and the particle generating the fringes may be confused with shattered fragments and be rejected by the inter-arrival time algorithm.

\subsection{Partially viewed ice branches}

Many ice particles develop branches extending from a few hundred micrometers up to $2-3 \mathrm{~mm}$ away from its center (i.e., bullet rosettes, dendrites and aggregated ice particles). The partially viewed branches of such particles could be confused with separate particles possessing short inter-arrival time (Fig. 2e), and be identified as artifacts. Rejecting images that are in contact with the edge of the array is one way to mitigate against this problem.

\subsection{Diffraction fringes}

Most particle imaging probes use coherent sources of light that result in the formation of diffraction fringes around the image of a particle. The binary representations of these fringes may manifest themselves as sparse disconnected pixel images that surround the main particle image. Such optical and imaging instrumentation effects may be confused with shattered fragments and result in identifying both diffraction fringes and the intact particle producing these fringes as artifacts.

For spherical particles such fringes become most pronounced when the dimensionless distance of the particles from the focal plane is close to $Z_{\mathrm{d}} \approx 1.9$ (Korolev, 2007; his Fig. 9) where $Z_{d}=\frac{4 \lambda Z}{D^{2}} ; \lambda$ is the wavelength; $Z$ is the distance from the object plane; $D$ is the particle diameter. Non-circular images produce diffraction fringes over a wider range of $Z_{\mathrm{d}}$. The probability of imaging the diffraction fringes increases with the increasing pixel resolution. Thus, for probes with coarse pixel resolution 100-200 $\mu$ m (e.g., HVPS, PIP, OAP-2DP), diffraction artifacts are quite rare, whereas for probes with $10-15 \mu \mathrm{m}$ pixel resolution (e.g., 2D-S, CIP) the effect of the diffraction fringes may have a significant effect on misidentification of intact particles as shattering artifacts. A few examples of diffraction fringes around the CIP out-of-focus images are shown in Fig. 3. These images were identified by the inter-arrival time algorithms as shattering artifacts and rejected. The 2-D data processing

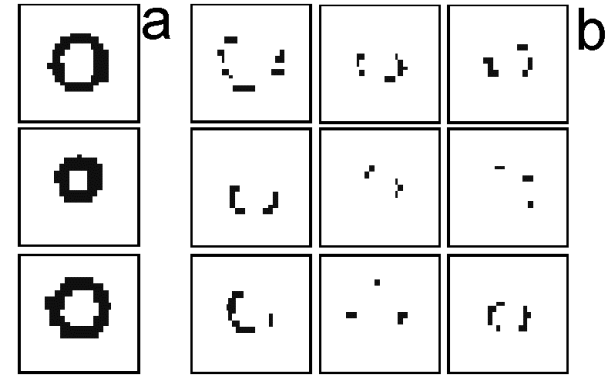

Figure 4. Examples of out-of-focus images measured by 2-D-S at $10 \mu \mathrm{m}$ pixel resolution. (a) Complete circle out-of-focus images; (b) fragmented out-of-focus images, which were registered in two or three image frames and identified as shattering artifacts by the interarrival time algorithm. The fragmented out-of-focus images are related to the particles passing through the sample volume near the edge of the depth-of-field.

software can be tuned to return large images to the pool of accepted images. However, setting the threshold for the sizes of the accepted images is ambiguous, and it may result in accepting shattering artifacts and rejecting intact particles.

\subsection{Out-of-focus fragmented images}

Out-of-focus images of particles traversing the sample area near the edges of the depth-of-field, when $Z_{\mathrm{d}}>6$ may appear as disconnected images (Korolev, 2007; Fig. 7). If the outof-focus fragmented image has a gap along the flight direction, it may be confused with a shattering artifact. Examples of the out-of-focus images, which were identified by ITA as shattered fragments, are shown in Fig. 4b. Out-of-focus images, such as images of transparent plates, quite often appear as fragmented and may also be identified by the ITA as artifacts.

\section{Results of measurements of inter-particle distances}

Because shattering generates particles by a very different physical process to those that occur naturally, the mode that describes the inter-arrival time distribution of these particles can be very different to that associated with the natural intact particles, which usually is well described by the Poisson distribution. This difference in the distribution of the particles manifests itself through differing inter-arrival time populations. Therefore, the distribution $\phi(\Delta t)$ can be used as one of the metrics for identifying shattering. The purpose of this section is to demonstrate the variety of $\phi(\Delta t)$ distributions and show their link to the particle size distributions. This consideration is expected to help further understanding of limitations of the ITA.

In order to reduce the effect of the air speed $u$ on $\Delta t$, the inter-particle distance $\Delta x=\Delta t / u$ will be used instead of $\Delta t$. Accordingly, the distribution $\phi(\Delta x)$ and the cut-off-distance 

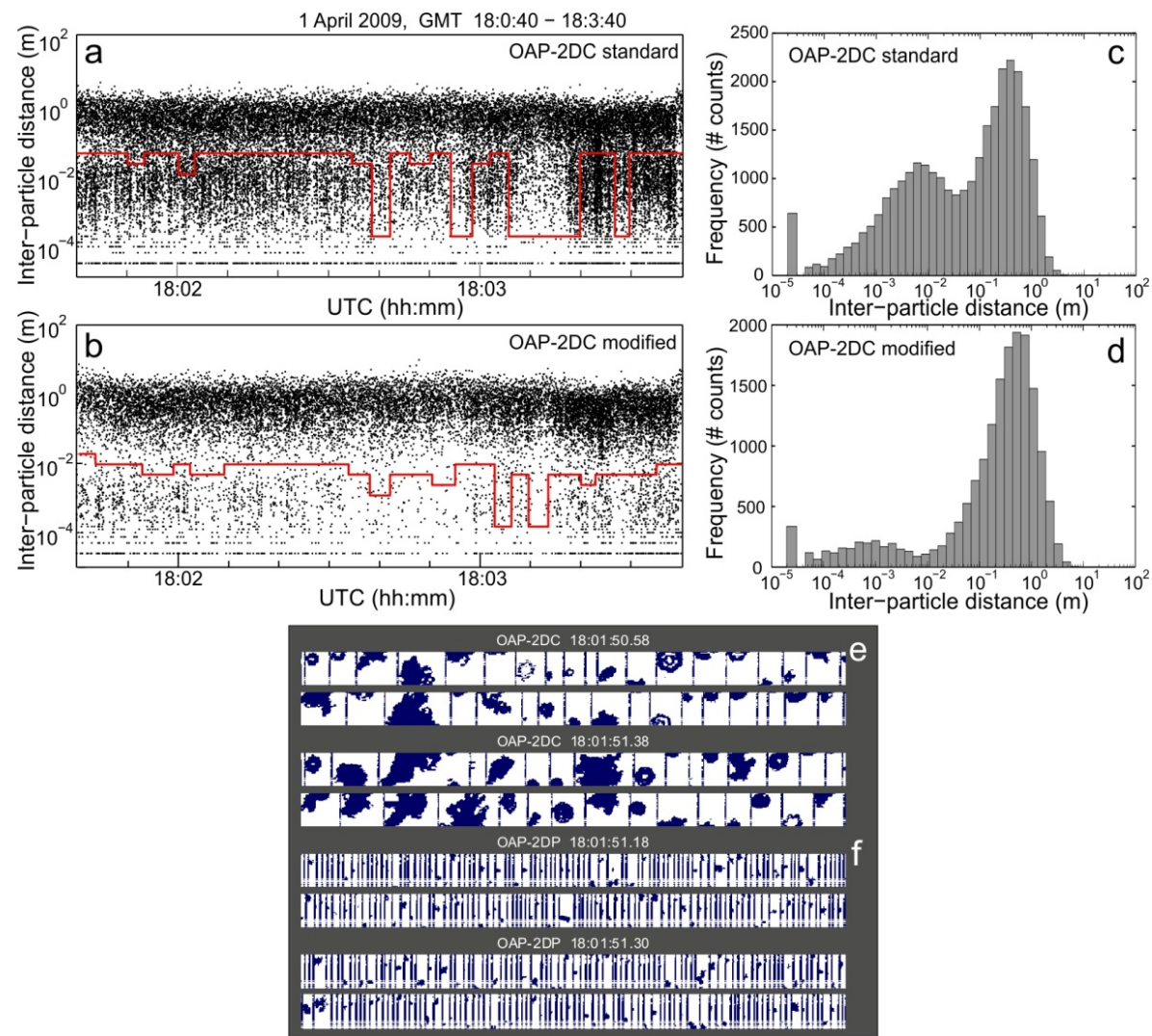

Figure 5. Comparison of inter-particle distances measured by standard (a) and modified (b) 2DC. Red lines in (a) and (b) indicate the cut-off distance $\chi^{*}$. The distribution of the inter-particle distances for standard (c) and modified (d) probes. Examples of images obtained with an OAP-2DC at $25 \mu \mathrm{m}$ pixel resolution (e) and an OAP-2DP at $200 \mu \mathrm{m}$ pixel resolution (f). The measurements were conducted on 1 April during an ascent through ice cloud from approximately 4600 to $5300 \mathrm{~m}$ in the Ottawa region. The temperature varied from -12 to $-17^{\circ} \mathrm{C}$.

$\chi^{*}=\tau^{*} / u$ will be used instead of $\phi(\Delta t)$ and $\tau^{*}$, respectively. We will also keep using the conventional term "interarrival time algorithm", although the term "inter-particle distance algorithm" would be more accurate.

\subsection{Description of the data set}

The data used here were collected during the Airborne Icing Instrumentation Evaluation Experiment (AIIE) flight campaign (Korolev et al., 2011, 2013b). The analysis of the inter-particle distance is focused on the measurements of two OAP-2DCs installed side-by-side in the NRC Convair580 aircraft. Both instruments have the same pixel resolution $25 \mu \mathrm{m}$, optics and electronics. However, one of the probes had the standard configuration, whereas the second one had modified arms with the K-tips installed (Korolev et al., 2013a). While K-tips still shatter ice particles, it has been demonstrated that they significantly mitigate the effect of shattering on ice particle measurements. Comparing measurements made with the standard and modified OAP-2DCs before and after applying the ITA provides an opportunity to assess the efficiency of the algorithm to successfully identify and filter out shattering artifacts. The 2-D data were aver- aged over 5-second time intervals. For most clouds sampled during the AIIE project such averaging provided statistically significant particle numbers to estimate the function $\phi(\Delta x)$ and cut-off-distance $\chi^{*}$. In the frame of this study the number of bins in $\phi(\Delta x)$ was selected to be 25 . This yields a reasonable compromise between the statistical significance of number of counts in each bin and the accuracy of finding $\chi^{*}$. Usually, for a typical shape of $\phi(\Delta x)$, a number of particle counts over 100 yielded an acceptable estimate of $\chi^{*}$ for the purposes of this work. A higher number of bins for $\phi(\Delta x)$ will require a higher number of counts, and therefore a longer averaging time.

\subsection{Examples of the inter-particle distance distribution}

The following three examples are based on the data collected during three different flights (1, 8 April 2009) and demonstrate how the presence of large particles and their concentration affect the inter-particle distance distribution $\phi(\Delta x)$. The first example demonstrates a moderate level of shattering and a high concentration of ice. The second example demonstrates more intense shattering and a low concentration of ice. 


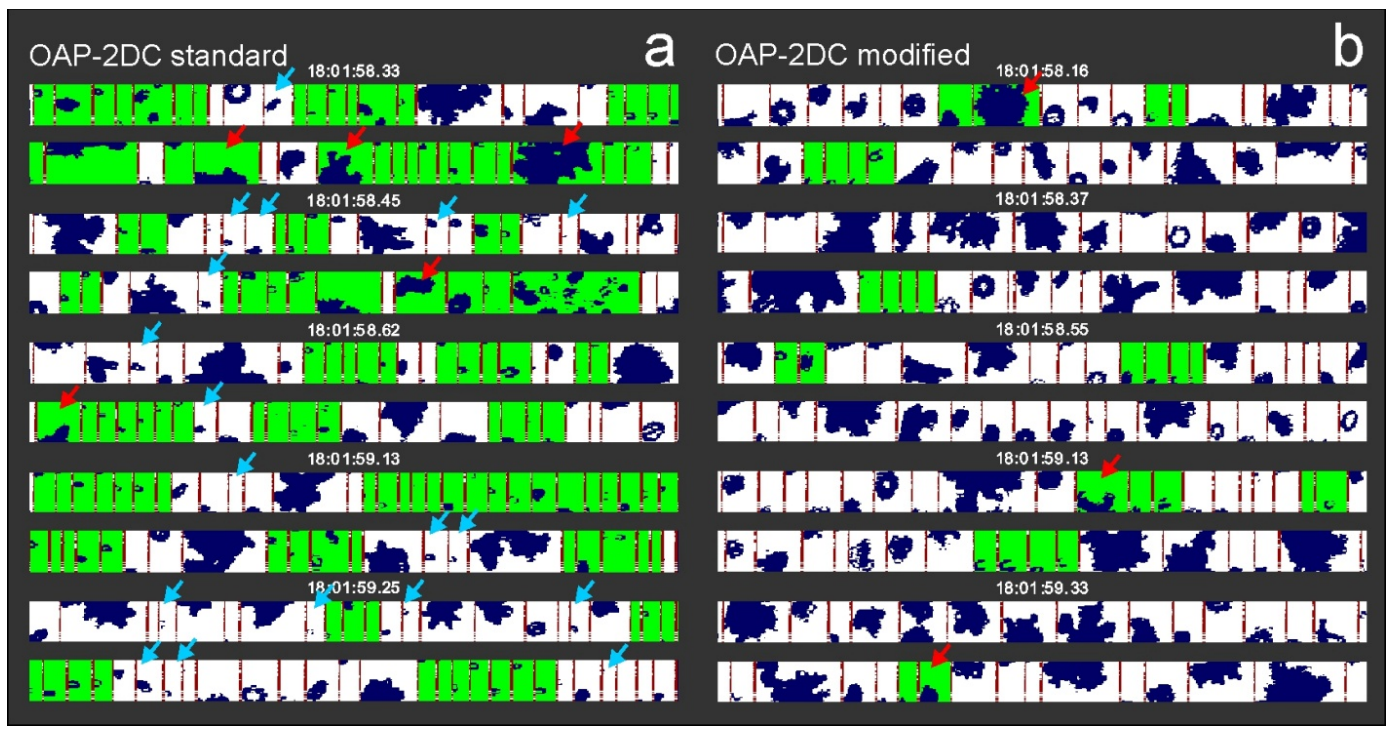

Figure 6. Examples of the results of the image rejection/acceptance processing with the inter-arrival time algorithm. The images of ice particles were simultaneously sampled by the standard (left) and modified (right) OAP-2DC during the time period shown in Fig. 5. Green backgrounds highlight the images identified by the inter-arrival time algorithm as artifacts. Images with a white background were accepted by the algorithm. As seen in (a), in some cases the standard OAP-2DC rejects large particles which appear to be intact (red arrows), at the same time it accepts particles which have the features of shattered fragments (blue arrows).

The last example demonstrates a case where shattering has a negligible effect.

\subsubsection{Overlapping modes}

Figure $5 \mathrm{a}$ and $\mathrm{c}$ show the time series of inter-particle distances measured by the standard and modified OAP-2DC in an ice cloud. Each inter-particle distance in Fig. 5a and b is represented by a dot. The red lines indicate the cut-offdistances. As seen from these two diagrams, the density of points below the red line is greater for the standard probe (Fig. 5a) than that for the modified one (Fig. 5b). The concentration measured by the modified 2DC, and corrected with the help of the ITA, varied from 20 to $80 \mathrm{~L}^{-1}$. Whereas, the uncorrected concentration measured by the standard 2DC varied from 300 to $1600 \mathrm{~L}^{-1}$. After applying corrections to the standard 2DC measurements its concentration varied in the range 150 to $700 \mathrm{~L}^{-1}$. The ice particle images measured by $2 \mathrm{DC}$ and 2DP are presented in Fig. 5e and f. Analysis of these images shows that the ensemble of ice particles was composed of two distinct habits: large spatial dendrites with sizes up to few millimeters and transparent plates with a characteristic size of a few hundred micrometers. The maximum particle size $D_{\max }$ calculated for each averaging time interval remained approximately constant and did not exceed $5 \mathrm{~mm}$.

The distributions of the inter-particle distances $\phi(\Delta x)$ calculated from the standard and modified 2DC probe data are shown in Fig. 5c and d. The inter-particle distance distribution, $\phi(\Delta x)$, for the standard 2DC displays a significant overlap between the long and short inter-arrival modes (Fig. 5c).
This may result in rejecting intact particles along with shattered artifacts when $\Delta x<\chi^{*}$, and, vice versa, accepting shattered fragments with intact particles for $\Delta x>\chi^{*}$.

The inter-particle distance distribution $\phi(\Delta x)$ for the modified probe has a relatively small overlap between the long and short distance modes, which suggests a better separation of shattered and intact particles. The number of particles associated with the short distance mode for the modified probe (Fig. 5d) is also reduced when compared to Fig. 5b. However, despite the larger separation between the short and long distance modes, the ITA still identifies large particles, which appear intact, as shattered fragments (e.g., Fig. 6b).

Figure 6 shows results from applying the ITA to the standard and modified OAP-2DC images sampled at the same time during one flight from the AIIE project. Images identified as shattered artifacts appear on a green background, whereas the accepted images have a white background. The number of rejected images for the standard probe (Fig. 6a) turned out to be nearly four times higher than that for the modified probe (Fig. 6b). Visual examination of the measurements made with the standard probe (Fig. 6a) reveals a number of images that we interpret as being intact (red arrows) but are rejected by the algorithm. Similar particle images can also be found in data for the modified probe (Fig. 6b), but there are far fewer images that look intact but are rejected. Some of the accepted images obtained by the standard probe we interpret as shattered artifacts (Fig. 6a, blue arrows). Shattered artifacts usually appear as elongated along the flight direction images due to the slower speed that they enter the sample volume. In many cases, the images of the shattering 
artifacts also have a hole in the center, that is the result of the greater likelihood of shatter products entering the sample volume closer to the arms and, consequently, far from the center of the depth-of-field. Further support for the assumption that the accepted images indicated by the blue arrows are shattered artifacts is provided by the absence of similar images recorded by the modified 2DC (Fig. 6b).

The apparently erroneous acceptance of the shattered artifacts and rejection of intact images in Fig. 6a is consistent with the large overlap between the short and long distance modes indicated in Fig. 5c. This example demonstrates that the ability of the ITA to segregate shattered artifacts and intact particles is reduced when the short and long distance modes become less separated.

One of the important goals of filtering out shattering artifacts is to obtain an accurate estimation of the PSD. Figure 7 shows the distributions of particle counts, concentration and mass calculated for all images before corrections, after corrections. These distributions were calculated for the image sizes measured along the photodiode array direction (i.e., perpendicular to the flight direction). Equivalent distributions are shown for the rejected images. During the image processing, the distribution of counts is used as a starting point for the following calculations of other distributions and bulk microphysical parameters. As seen from Fig. 7a and $\mathrm{d}$ for both standard and modified OAP-2DCs the number of small particles rejected by the inter-arrival algorithm is nearly two orders of magnitude greater than for the large particles. This is consistent with the concept that shattered particles are mainly composed of small fragments. Experimental studies indicate that the fragments of ice particles shattered at the aircraft speed have characteristic size from tens to hundreds of micrometers (Vidaurre and Hallett, 2009). Therefore, it is hypothesized that rejected particles larger than approximately $1 \mathrm{~mm}$ (Fig. 7 ) are likely related to the cases depicted in Fig. 2b, d, e and $f$.

It is interesting to note that for the modified probe the ITA corrected and uncorrected distributions agree to better than $10 \%$ for particles larger $600 \mu \mathrm{m}$ (in Fig. $7 \mathrm{~d}-\mathrm{f}$ ). However, for the standard probe the separations between ITA corrected and uncorrected distributions remain approximately constant for $D>600 \mu \mathrm{m}$ (Fig. $7 \mathrm{a}-\mathrm{c}$ ) and it varies from 20 to $30 \%$. Despite the rejection of a large fraction of small particles (Fig. 7b) in the modified probe, the concentration of small particles $(D<200 \mu \mathrm{m})$ is only reduced after correction by a factor of 2 to 3 (Fig. 7e). At the same time, the concentration of particles in the small size bins $(D<200 \mu \mathrm{m})$ for the standard probe after ITA correction still remains higher than for the modified probe. This is consistent with the above conclusion that the ITA is unable to filter out all shattering artifacts. The corrected concentration of small particles $(D<200 \mu \mathrm{m})$ measured by the modified probe is still high. It is difficult to conclude whether these particles are real or associated with shattering artifacts, for instance due to singletons, or other mis-sizing and concentration errors.
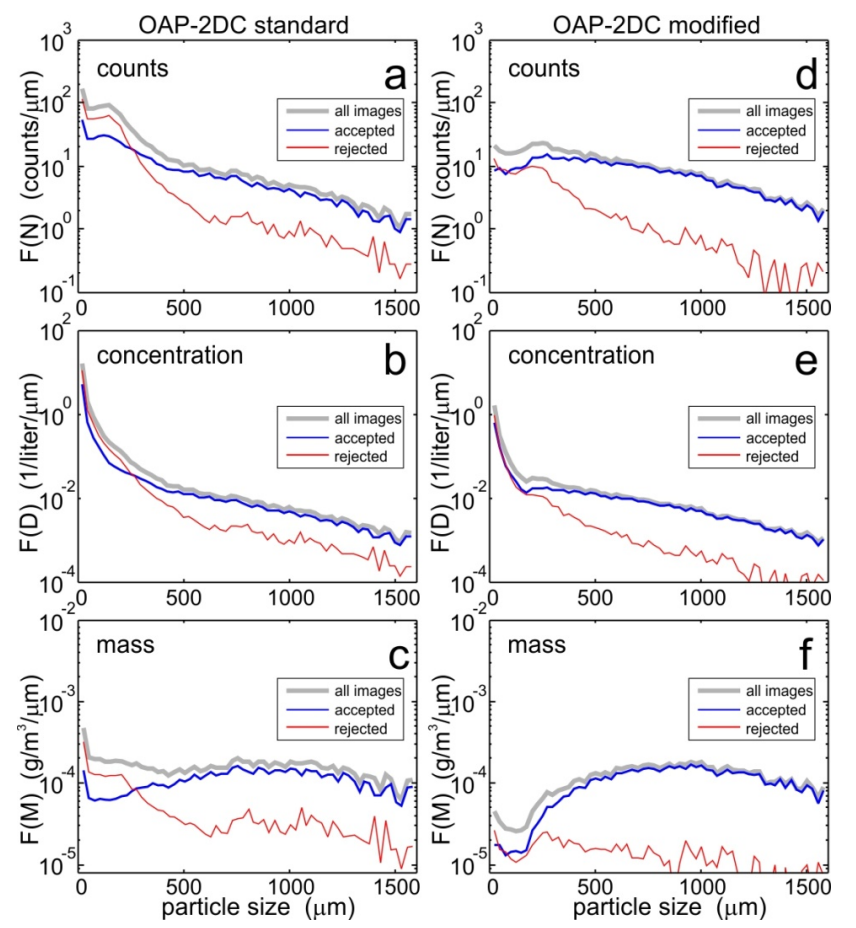

Figure 7. Distributions of particle counts (a, d), concentration (b, e), and mass (c, f) calculated for all images (grey), and accepted (blue) and rejected (red) identified by the inter-arrival time algorithm. The distributions were calculated for the standard (left) and modified (right) OAP-2DCs data collected in the cloud shown in Fig. 5.

Comparisons of corrected mass distributions for standard and modified probes in Fig. 7c and $\mathrm{f}$ show a large difference between them for $D<500 \mu \mathrm{m}$. However, since large particles are the major contributors into the total mass, the discrepancy at the small size end of the PSD does not produce any significant effect on the bulk IWC. Integration of the mass distributions for standard and modified probes shows that IWC corrected standard is systematically higher than that for the modified OAP-2DCs. For this particular case, IWC corrected standard is approximately $20 \%$ higher, and the mean IWC values averaged over the entire time interval is approximately $4 \%$ higher than the modified OAP-2DC.

\subsubsection{Large particles}

Figure 8 shows the changes of inter-particle distances and distributions $\phi(\Delta x)$ measured during a descent through precipitating aggregates of dendrites with $D_{\max }$ ranging from 0.5 to $1.5 \mathrm{~cm}$. The total number of particle counts per second in the modified probe varied from 10 to 70 and the rate of counts for the standard probe varied in the range 100 to 300 . The ITA corrected concentration measured by the modified OAP-2DC varied from 0.5 to $5 \mathrm{~L}^{-1}$, whereas the uncorrected concentration measured by the standard probe varied from 100 to $300 \mathrm{~L}^{-1}$. After applying corrections to the standard 

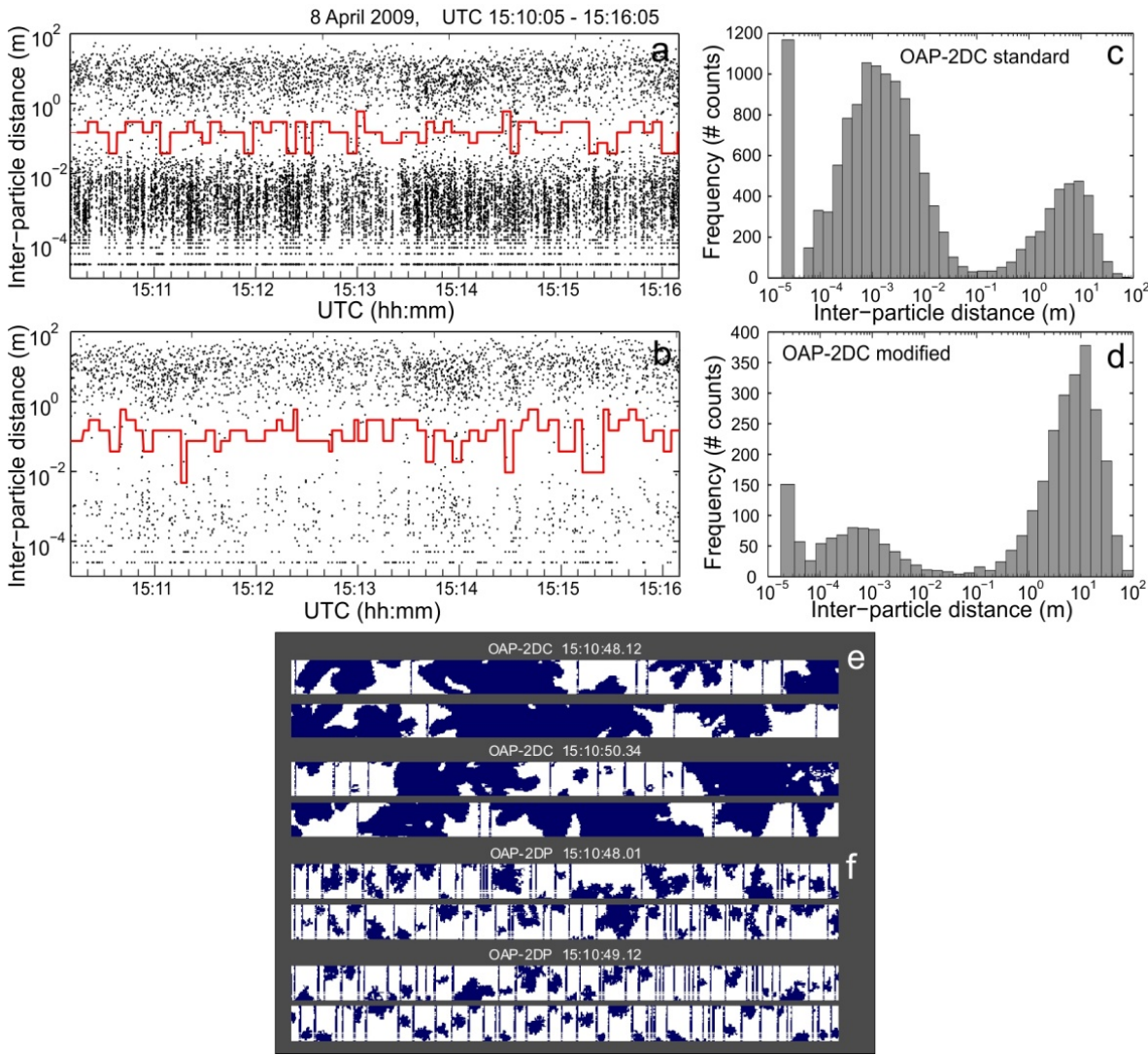

Figure 8. Comparison of inter-particle distances measured by standard (a) and modified (b) 2DC. Red lines in (a) and (b) indicate the cut-off distance $\chi^{*}$. The distribution of the inter-particle distances for standard (c) and modified (d) probes. Examples of images obtained with an OAP-2DC at $25 \mu \mathrm{m}$ pixel resolution (e) and an OAP-2DP at $200 \mu \mathrm{m}$ pixel resolution (f). The measurements were conducted during on 8 April (1st flight) during descent through precipitating dendrites from approximately 1300 to $500 \mathrm{~m}$ in the Ottawa region. The temperature varied from -8 to $-2{ }^{\circ} \mathrm{C}$.

2DC data its concentration varied in the range 1 to $10 \mathrm{~L}^{-1}$. The appearance of the particle images measured by OAP2DC and OAP-2DP are shown in Fig. 8e and f.

The distinctive feature of the inter-particle spatial distribution $\phi(\Delta x)$ calculated for the standard OAP-2DC is that it has an exceptionally high number of counts $(88 \%)$ associated with the short distance mode. This indicates that the standard probe observed mostly shattering artifacts. In contrast, for the modified probe the distribution $\phi(\Delta x)$ the number of counts in the short distance mode is smaller than for the long distance mode.

The results of segregating the intact particles and shattered artifacts for standard and modified OAP-2DC performed by the ITA are shown in Fig. 9. The measurements made with the standard probe are dominated by artifacts with very few accepted images (Fig. 9a). The particle imagery obtained with the modified probe is largely devoid of the small images typically associated with shattered fragments (Fig. 9b). An absence of small particles in subsaturated precipitating regions is consistent with the commonly accepted concept of ice formation. It is worth noting that a few small accepted images (indicated by the blue arrows) still appear in the standard 2DC imagery in Fig. 9a. It is possible that these small images are related to single fragments as in Fig. $2 \mathrm{c}$ and were misidentified by the ITA as intact particles.

Despite the fact that most of the images in Fig. 9b appear to be intact dendrites, many of them were rejected. Visual inspection of the rejected images in Fig. 9b indicates that nearly all of them are partially viewed images. It is likely that the partially viewed branches of dendrites (e.g., Fig. 2e) has led to the ITA confusing the partially observed closely spaced arms of the dendrite with the shattered artifacts (Sect. 3.4).

The results shown in Fig. 9 demonstrate that even for the cases where there is a good separation of the short and long distance modes (Fig. 8c, d) the ITA still misidentifies shattering artifacts and intact particles.

The distributions of particle counts, concentration and mass obtained with the standard and modified probes are shown in Fig. 10. Figure 10a and d demonstrate that the number of counts of small particles is much greater for the standard probe than for the modified probe. This is consistent with the 2-D images shown in Fig. 9. The ITA identified 


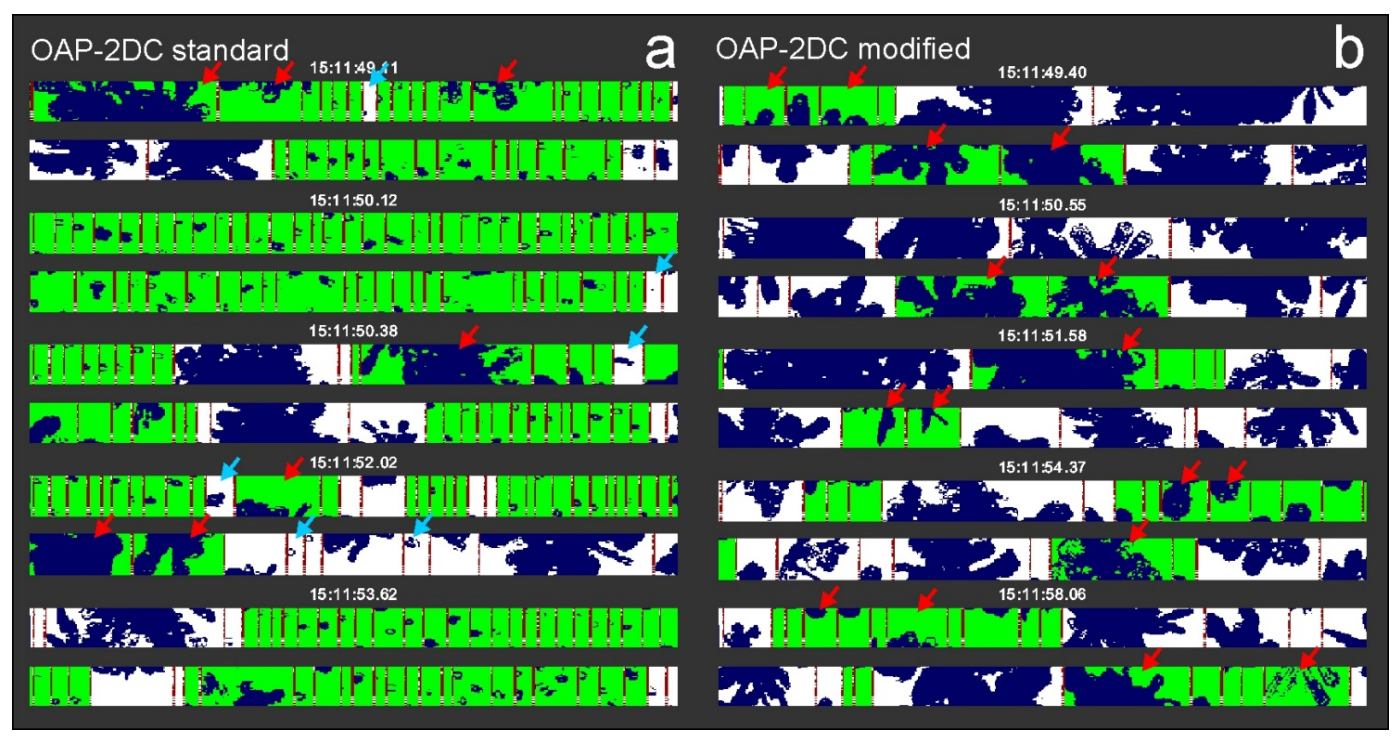

Figure 9. Examples of the results of the image rejection/acceptance processing with the inter-arrival time algorithm. The images of ice particles were simultaneously sampled by the standard (left) and modified (right) OAP-2DC during the time period shown in Fig. 8. A green background highlights the images identified by the inter-arrival time algorithm as artifacts. Images with a white background were accepted by the algorithm. As seen in (a), in some cases the standard OAP-2DC rejects large particles which appear to be intact (red arrows), at the same time it accepts particles which have the features of shattered fragments (blue arrows).
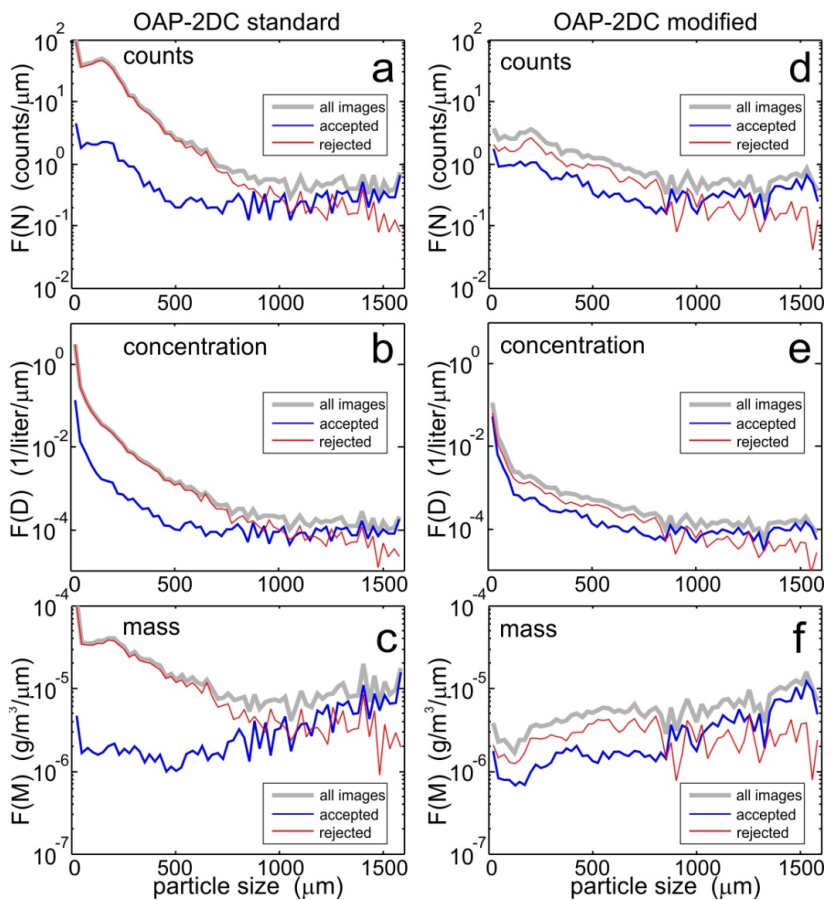

Figure 10. Distributions of particle counts (a, d), concentration (b, e), and mass (c, f) calculated for all images (grey), and accepted (blue) and rejected (red) identified by the inter-arrival time algorithm. The distributions were calculated for the standard (left) and modified (right) OAP-2DCs data collected in the cloud shown in Fig. 8. most of the small image counts as artifacts (Fig. 10a). However, the number of small images accepted for the standard probe is still greater than the modified probe (Fig. 10d). The same conclusion applies to the concentration and mass distributions shown in Fig. 10b, c and Fig. 10e, f.

We note that the shape of rejected distributions for the modified probe is very similar to the shape of the uncorrected distributions (Fig. 10d, e, f). Furthermore, the distributions of the accepted and uncorrected images approach each other only for particle sizes $D>1500 \mu \mathrm{m}$ (Fig. 10a, b, c), in contrast to the case shown in Fig. 7. For both probes, the number of rejected large particles remains relatively large, and it is related to misidentifying large particles as shattered artifacts.

\subsubsection{Small ice particles}

The next example was obtained during a sampling of cirrus clouds at a temperature of $-35^{\circ} \mathrm{C}$ and altitude of $7500 \mathrm{~m}$. The maximum size of particles varied from 200 to $400 \mu \mathrm{m}$, and did not exceed $500 \mu \mathrm{m}$. The particle concentration measured by the standard and modified OAP-2DCs agreed well and varied from 20 to $180 \mathrm{~L}^{-1}$.

Figure 11 shows the inter-particle spacings (Fig. 11a, b) and their distributions $\phi(\Delta x)$ for the standard and modified probes. As can be seen in Fig. 11c and d, the interparticle spacing distribution $\phi(\Delta x)$ is monomodal for both probes. Since the ITA used here is most efficient only for the cases with bimodal distributions, for this particular case with monomodal $\phi(\Delta x)$, the fraction of rejected particles for both $2 \mathrm{DCs}$ is quite small and does not exceed $0.7 \%$. This is 


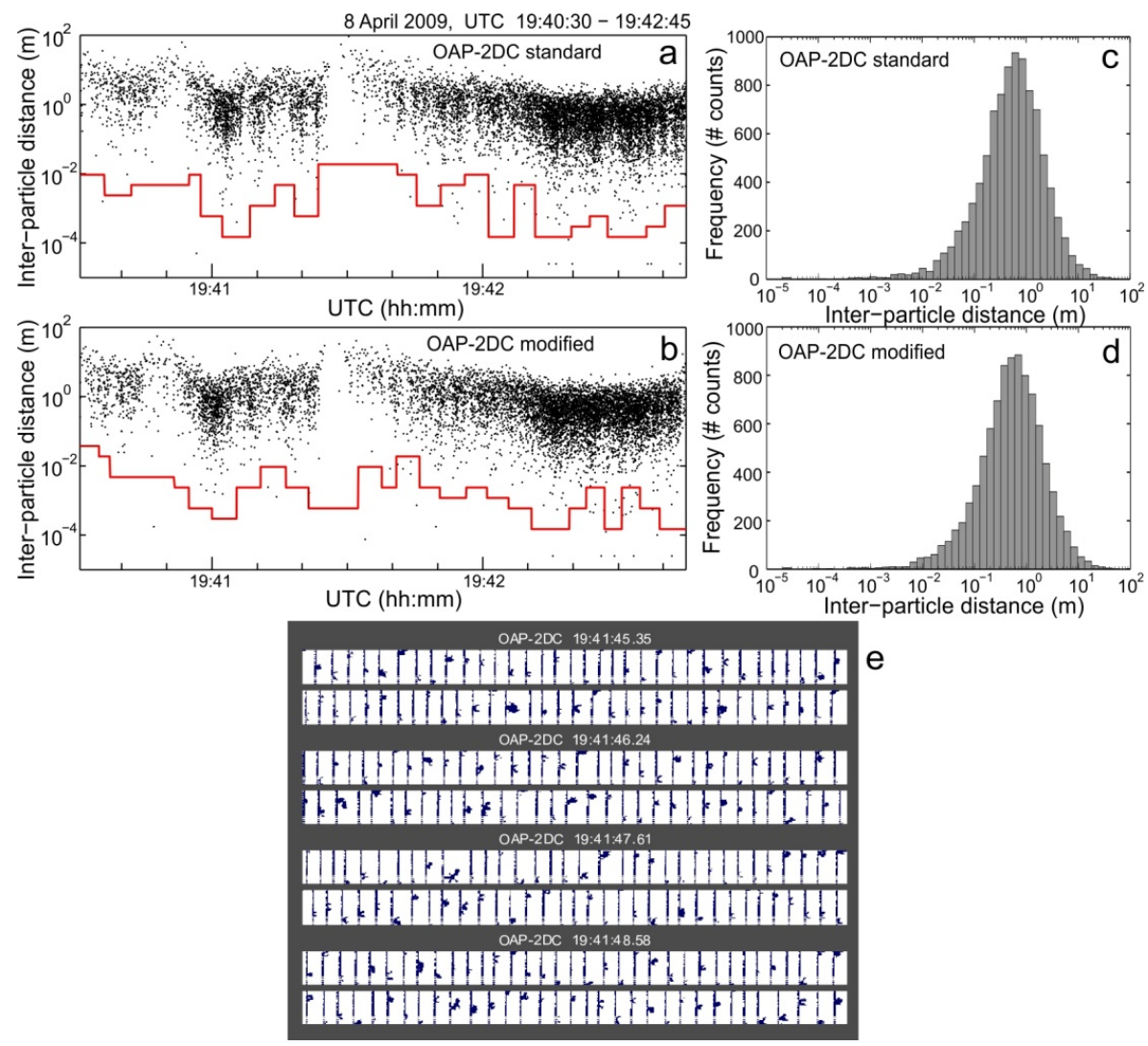

Figure 11. Same as in Fig. 8. The measurements were collected on 8 April (2nd flight) in cirrus clouds at altitude $7500 \mathrm{~m}$ and temperature $-35^{\circ} \mathrm{C}$.

demonstrated by the small number of points that lie below the cut-off-distance line (red) in Fig. 10a and b (for which $\Delta x<\chi^{*}$ ), and that Fig. 12 shows very few images identified by the ITA as artifacts.

The distributions of particle counts, concentration and mass are shown in Fig. 13. All three distributions obtained with the standard and modified probes agree well with each other. The fraction of rejected artifacts is small and for practical purposes their effect on the size and mass distributions has negligible effect.

These results show that for this specific case there is not much difference between the measurement obtained by the standard and modified probes. This suggests that for the cases where the ice PSD is narrow $\left(D_{\max }<400 \mu \mathrm{m}\right)$ the effect of shattering on the standard OAP-2DC measurements is quite small and can be neglected. This finding also implies that there exists a threshold size below which shattering of ice crystals does not produce any significant effect on measurements. This will likely vary according to instrumental and microphysical characteristics. Of course, even though the probes agree for this case, problems relating to mis-sizing and concentration errors for particles smaller than $\sim 100$ microns still exist (Korolev et al., 1998; Strapp et al., 2001).

\subsection{Statistical characteristics of inter-particle distances in shattering events}

Figures 5a, b, 8a, b and 11a, b contain a plethora of information about the statistical characteristics of shattering events and their effect on ice particle measurements. This data can also be used in the development of future algorithms for the data processing and numerical simulations of the shattering effects. In this section some of the statistical characteristics of shattering processes will be investigated and compared with microphysical metrics.

Figure 14 shows distributions of the number of particles $N_{\mathrm{s}}$ within each shattering event (a1-a3), distributions of the length of spatial clusters along the flight direction $L_{\mathrm{s}}$ (b1-b3), distributions of distances between shattering events $L_{i}(\mathrm{c} 1-\mathrm{c} 3)$, and the number of particles between shattering events $N_{i}(\mathrm{~d} 1-\mathrm{d} 3)$. These distributions were calculated for the cloud regions shown in Figs. 5, 8 and 11.

The distribution $f\left(N_{\mathrm{s}}\right)$ (Fig. 14a1-a3) for both standard and modified probes is well represented by an exponential function (Field et al., 2003). This suggests that the statistics of shattered fragments passing through the sample volume can be approximated by the Poisson distribution. Analysis of the other cases indicates that the slope of the distribution 


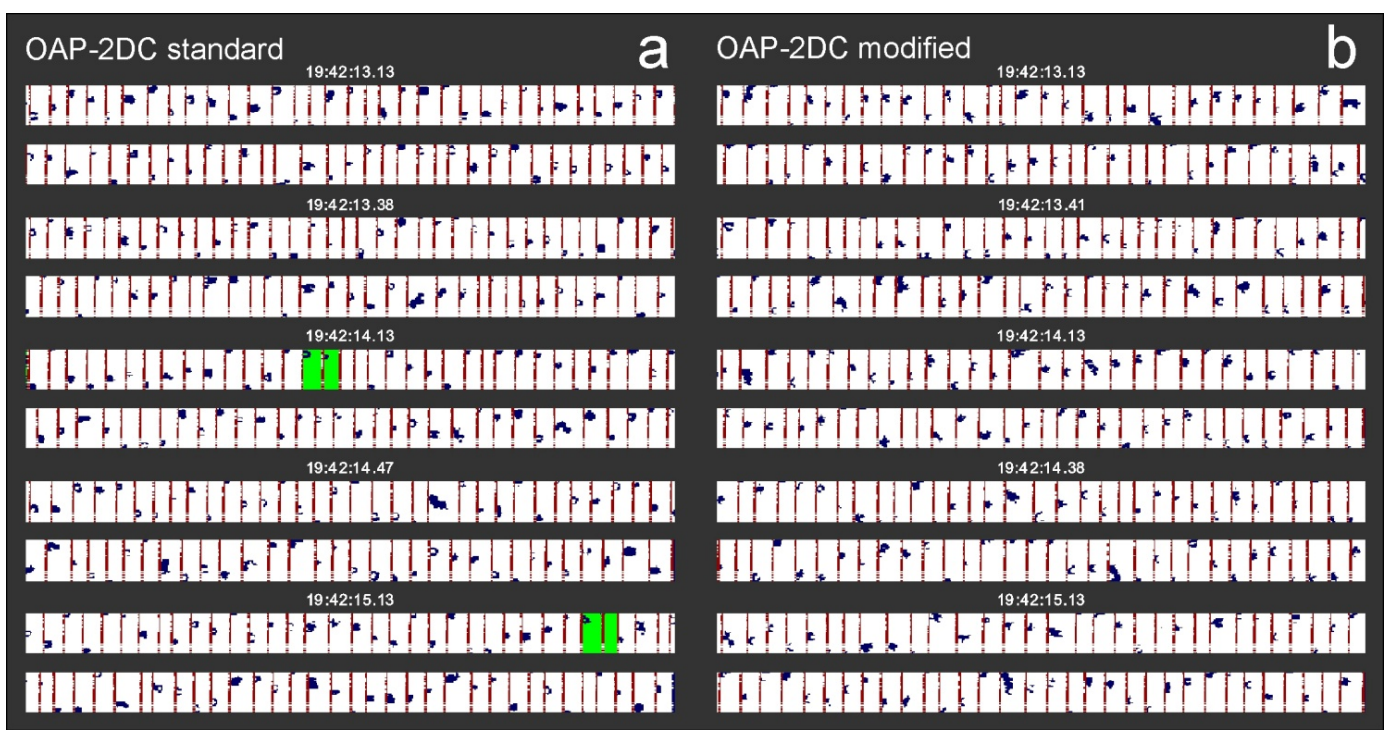

Figure 12. Same as in Fig. 6. The measurements of the images were obtained during the time period shown in Fig. 11.
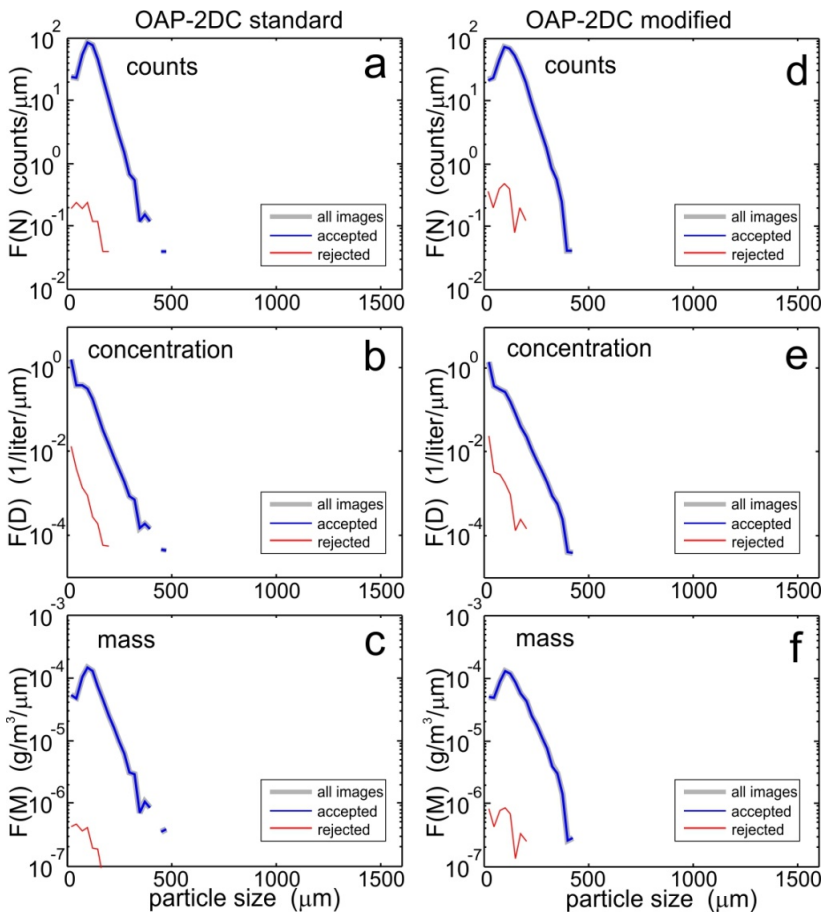

Figure 13. Distributions of particle counts $(\mathbf{a}, \mathbf{d})$, concentration $(\mathbf{b}$, e), and mass (c, f) calculated for all images (grey), and accepted (blue) and rejected (red) identified by the inter-arrival time algorithm. The distributions were calculated for the standard (left) and modified (right) OAP-2DCs data collected in the cloud shown in Fig. 11.

depends on the presence of large particles in the size distribution and is correlated with $D_{\max }$ such that increasing $D_{\max }$ leads to a shallower slope for $f\left(N_{\mathrm{s}}\right)$ : Figure $14 \mathrm{a} 2$ displays the shallowest slope in $f\left(N_{\mathrm{s}}\right)$ for the case with the greater $D_{\max }$.

The distribution of $f\left(N_{\mathrm{s}}\right)$ monotonically increases with decreasing $N_{\mathrm{s}}$, having a maximum for events with two shattered fragments. Extrapolating $f\left(N_{\mathrm{s}}\right)$ towards 1, i.e., shattering events with one particle, suggests that the number of singletons may be quite high. Existence of single particle shattering events presents a principal limitation of the interarrival algorithm, since such particles cannot be unambiguously identified as artifacts merely based on the analysis of $\Delta x$.

The maximum number of the shattered fragments in a shattering event measured by the standard OAP-2DC during the AIIE project reached 60. Whereas for the modified probe, the maximum number of fragments for this data set was found to be 14 . Figure $14 \mathrm{a} 1$ and a 2 also demonstrate that the modified OAP-2DC on average has a smaller number of fragments per shattering event, and therefore the antishattering tips can efficiently mitigate shattering.

The density function of the length of spatial clusters of shattered fragment $f\left(L_{\mathrm{s}}\right)$ can also be well approximated by an exponential function (Fig. 14b1, b2). Clusters with a short length have the highest probability and they are associated with two-particle shattering events. The spatial length of the shattering clusters is presented by a cascade of scales ranging from zero to tens of centimeters. The maximum length of the shattered clusters measured by the standard and modified OAP-2DCs during the AIIE project reached 30 and $3 \mathrm{~cm}$, respectively.

The density functions of distances between shattered events $f\left(L_{i}\right)$ are shown in Fig. 14c1-c3. The characteristic scale of $L_{i}$ is determined by the concentration of particles capable of generating shattering events. For example, the case 

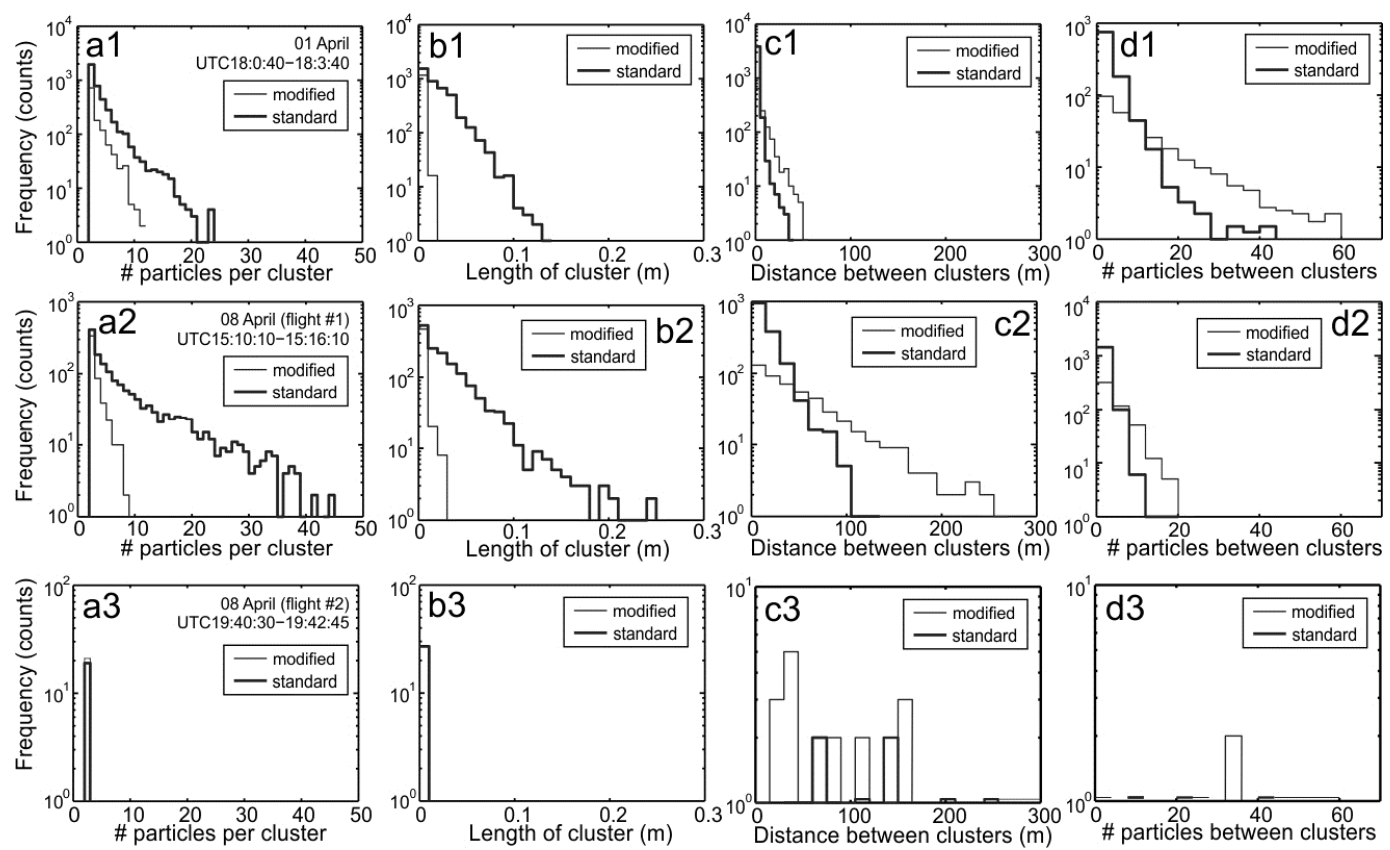

Figure 14. Distributions of number of particles per shattering event (a1, a2, a3), length of the clusters of shattered artifacts along the flight direction $(\mathbf{b 1}, \mathbf{b 2}, \mathbf{b 3})$, distance between the clusters with shattered fragments $(\mathbf{c 1}, \mathbf{c 2}, \mathbf{c 3})$, number of intact particles between shattered events (d1, d2, d3) calculated for the cases shown in Fig. 5 (top row), Fig. 8 (middle row) and Fig. 11 (bottom row), respectively.

in Fig. 14c3 has the highest total concentration of ice particles. However, only $0.7 \%(\sim 30$ counts $)$ of them generate shattering events. As a result the $f\left(L_{i}\right)$ in Fig. $14 \mathrm{c} 3$ has a statistically insignificant distribution. As in the previous cases $f\left(L_{i}\right)$ can be well approximated by an exponential function and the characteristic value of $L_{i}$ for modified OAP-2DC is larger than that for the standard probe (Fig. 14c1, c2).

The behavior of the function $f\left(N_{i}\right)$ in Fig. $14 \mathrm{~d} 1-\mathrm{d} 3$ is very similar to that of $f\left(L_{i}\right)$ (Fig. 14c1, c2, c3). For the case depicted in Fig. 14d 3 the low number of shattering events mean that most of the counts are outside of the scale.

Figure 15 shows the distributions of cut-off distances, $f\left(\chi^{*}\right)$, for the standard and modified OAP-2DCs averaged over all ice clouds sampled during the AIIE project. Because the value of $\chi^{*}$ depends of the size distribution of ice particles and their habits, the shape of the distribution, $f\left(\chi^{*}\right)$, will be determined by a combination of cloud characteristics, aircraft and instrument properties. However, the distributions of $f\left(\chi^{*}\right)$ in Fig. 15 allow a few conclusions to be made. Firstly, for the OAP-2DC $\chi^{*}$ can vary from tens of micrometers to approximately one meter. Secondly, the standard probe $\chi_{\mathrm{st}}^{*}$ has a mode at approximately $10 \mathrm{~cm}$, whereas the modified probe $\chi_{\mathrm{mdf}}^{*}$ has a mode at approximately $2 \mathrm{~cm}$.

\subsection{Effect of particle sizes}

The effect of particle sizes on shattering is demonstrated in Fig. 16 which shows the maximum number of fragments per shattering event $N_{\text {smax }}$ vs. $D_{\max }$ for standard and modified probes. The $N_{\text {smax }}$ and $D_{\max }$ were calculated for each $5 \mathrm{~s} \mathrm{av-}$ eraging interval for the entire AIIE project. Figure 16a shows that for $D_{\max }<15 \mathrm{~mm}, N_{\text {smax }}$ correlates well with $D_{\max }$ and therefore the dependence $N_{\text {smax }}\left(D_{\max }\right)$ can be parameterized with a linear function (Fig. 16a). However, for the modified probe the correlation coefficient between $N_{\text {smax }}$ and $D_{\max }$ is low (0.57) and the $N_{\text {smax }}\left(D_{\max }\right)$ saturates at $N_{\text {smax }} \sim 15$ when $D_{\max }>5 \mathrm{~mm}$ (Fig. 16b).

Figure 17 shows the maximum length of shattering clusters $L_{\text {smax }}$ vs. $D_{\max }$ for standard and modified probes. Similar to the case in Fig. 16, $L_{\text {smax }}$ and $D_{\max }$ were calculated for each $5 \mathrm{~s}$ averaging interval. A relatively high correlation coefficient between $L_{\mathrm{smax}}$ and $D_{\max }(0.78)$ for the standard probe allows linear parameterization of $L_{\mathrm{smax}}\left(D_{\max }\right)$ (Fig. 17a). The correlation coefficient between $L_{\text {smax }}$ and $D_{\max }$ for the modified probe is quite low (0.29), but $L_{\mathrm{smax}}$ is never longer than $3 \mathrm{~cm}$ for all $D_{\max }$ encountered.

The nearly linear increase of $N_{\text {smax }}$ and $L_{\text {smax }}$ with the increase of $D_{\max }$ for the standard probe (Figs. 16a, 17a) indicates a strong dependence of particle size on particle shattering.

\section{Monte Carlo simulation of inter-particle distance function}

The statistical parameters obtained from the shattering analysis were used to constrain Monte Carlo simulations of the effect of shattering on particle measurements. These simula- 

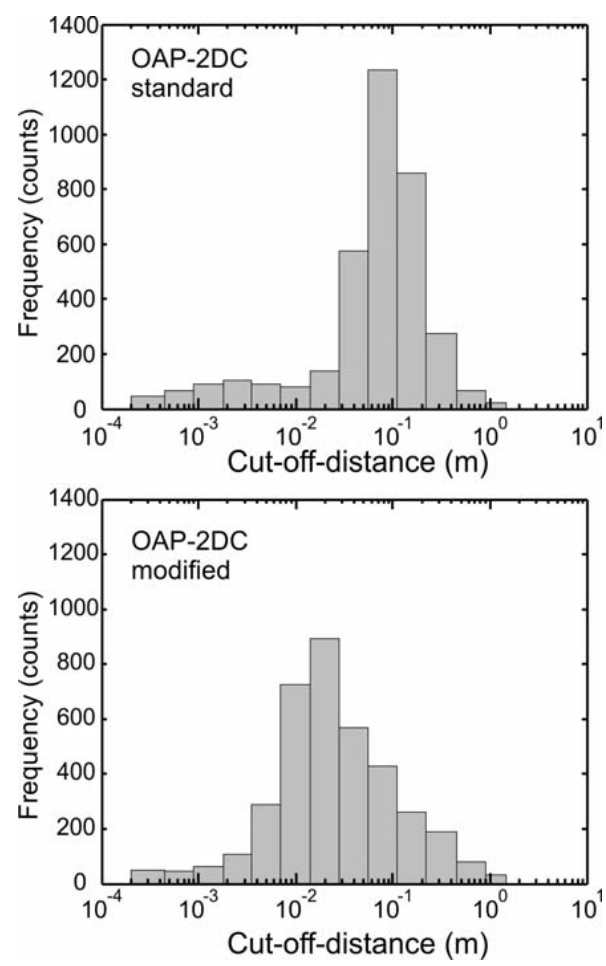

Figure 15. Distribution of cut-off distances $\chi^{*}$ for standard (a) and modified (b) OAP-2DCs averaged over all ice clouds sampled during the AIIE project.

tions were used to understand the influence of various parameters on the shape of the inter-particle distance distribution, $\phi(\Delta x)$.

For each simulation, two fluxes of particles with the same concentration $n_{0}$ are assumed to interact with the probe. The first flux represents intact particles and passes through the sample area $S_{0}$ of the probe. The second flux represents the shattered particles and passes through a "shattering" area for the probe, $S_{\mathrm{sh}}$, rebounds and subsequently passes through $S_{0}$. After passing though $S_{\mathrm{sh}}$, a particle breaks down into $N_{\text {sh }}$ fragments.

The inter-particle distance between the intact particles was simulated assuming a Poisson distribution by combining a random number generator with an exponential distribution with mean distance $\bar{\chi}_{0}=\frac{n_{0}}{S_{0}}$. The inter-particle distance between the shattering events was also simulated using an exponential distribution with mean distance $\bar{\chi}_{\mathrm{ev}}=\frac{n_{0}}{S_{\mathrm{sh}}}$. Based on the results obtained in Sect. 4.3, the number of shattered fragments was simulated by a random number generator with exponential distribution with average $\bar{N}_{\text {sh }}$. The distance between the shattered fragments in each shattered event was also simulated by the exponential distribution with average $\bar{\chi}_{\mathrm{sh}}=\frac{\bar{L}_{\mathrm{s}}}{\bar{N}_{\mathrm{sh}}}$, where $\bar{L}_{\mathrm{s}}$ is the average distance of the cluster of shattered fragments. After sorting arrival times, the two flows were merged together to form a time series of intact particles mixed with the shattering artifacts.
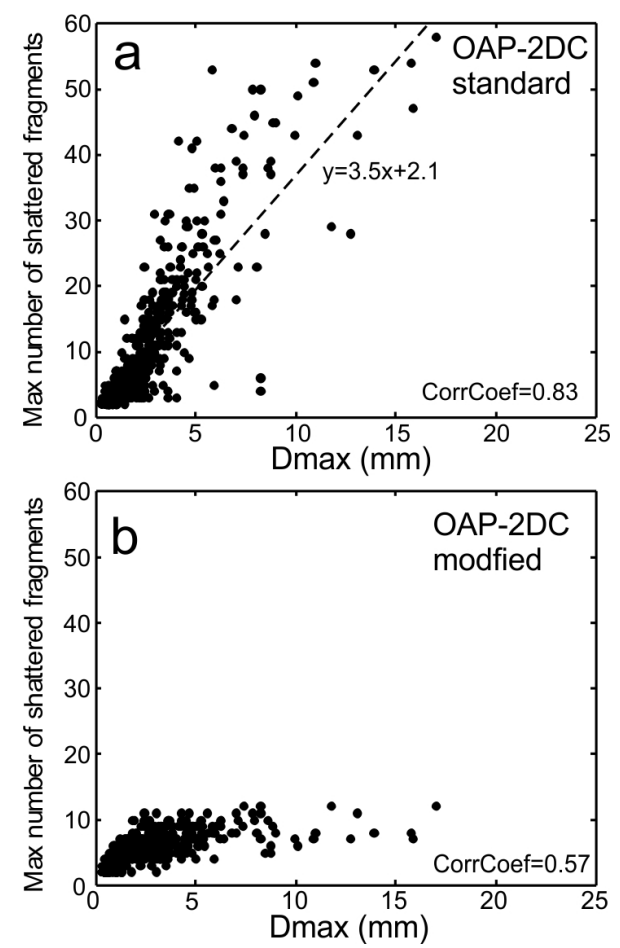

Figure 16. Scatter diagram of the maximum number of shattered fragments per event vs. maximum particle sizes for standard (a) and modified (b) OAP-2DCs for all ice clouds sampled during the AIIE project.

The modeling results presented below were performed for $S_{0}=50 \mathrm{~mm}^{2}, S_{\mathrm{sh}}=5 \mathrm{~mm}^{2}, \bar{L}_{\mathrm{s}}=1 \mathrm{~cm}$ and $\bar{N}_{\mathrm{sh}}=5$. The particle size distribution was assumed to be monodisperse.

Figure 18 shows the modeled distributions of the interparticle distances for intact particles only, $\phi_{\mathrm{i}}(\Delta x)$ (blue), shattered particles only, $\phi_{\mathrm{S}}(\Delta x)$ (red), and all particles that passed through the sample volume, $\phi(\Delta x)$ (black). The distribution $\phi_{\mathrm{i}}(\Delta x)$ represents the Poisson process and has a single mode, whereas $\phi_{\mathrm{S}}(\Delta x)$ has two modes. The long distance mode in $\phi_{\mathrm{S}}(\Delta x)$ is determined by the characteristic distance between the shattered events $\bar{\chi}_{\mathrm{ev}}$, whereas the short distance mode is associated with the characteristic distances between the shattered fragments $\bar{\chi}_{\mathrm{sh}}$, which passed through the sample volume. The long distance mode in $\phi_{\mathrm{s}}(\Delta x)$ also includes single particle shattering events. This is what we see for the long mode of the red line (rebounders/singletons) and the blue line. Both of these are continuous processes, whereas the shorter shattering mode is intermittent conditional on a collision occurring in the shattering volume. It is important to mention that the inter-particle distribution $\phi(\Delta x)$ representing the flow of all particles cannot be presented as an algebraic superposition of $\phi_{\mathrm{s}}(\Delta x)$ and $\phi_{\mathrm{i}}(\Delta x)$. In this regard, the conceptual diagrams in Fig. 1 show oversimplified inter-arrival time distributions, which help understanding, but do not reflect the actual shape of $\phi_{\mathrm{i}}(\Delta x)$. 

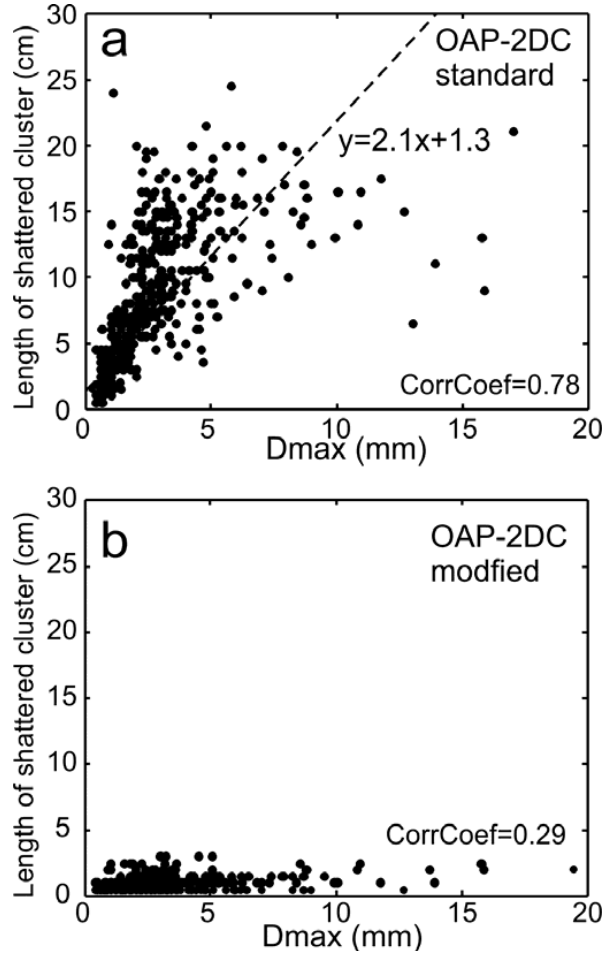

Figure 17. Scatter diagram of the length of clusters of shattered fragments vs. maximum particle sizes for standard (a) and modified (b) OAP-2DCs for all ice clouds sampled during the AIIE project.

Figure 19 shows four distributions $\phi(\Delta x)$ calculated for the particle concentrations $1,10,100$, and $1000 \mathrm{~L}^{-1}$. Figure 19 shows that the long distance mode approaches the short distance mode, when the particle concentration $n_{0}$ increases. However, the short distance mode is insensitive to the changes of the particle concentration, and it remains at the same position, when $n_{0}$ increases (Fig. 19a, b, c). The location of the cut-off-distance $\chi^{*}$ appears insensitive to the changes in $n_{0}$, remaining approximately constant.

For increasing $n_{0}$ the reduced separation of the long and short distance mode results in an increased overlap of the distributions associated with these modes. As described above, increasing the overlap of these distributions reduces the efficiency of the ITA to segregate intact particles and shattered artifacts. At high concentrations the short and long distance modes merge, resulting in the vanishing of the inter-modal minimum. At that stage the ITA becomes much less efficient or even disabled (e.g., Fig. 19d).

In the above simulations the particle size distribution was assumed to be monodisperse. One of the consequences of this assumption is that all of the particles possessed the same shattering efficiency and $\bar{N}_{\text {sh }}$ remains the same for all particles. In reality, particle sizes in natural clouds are represented by broad distributions. As indicated above, $\bar{N}_{\text {sh }}$ depends on particle sizes and that for small particles with $D<400 \mu \mathrm{m}$ $\bar{N}_{\mathrm{sh}} \rightarrow 0$. Therefore, the concentration $n_{0}$ should be inter-

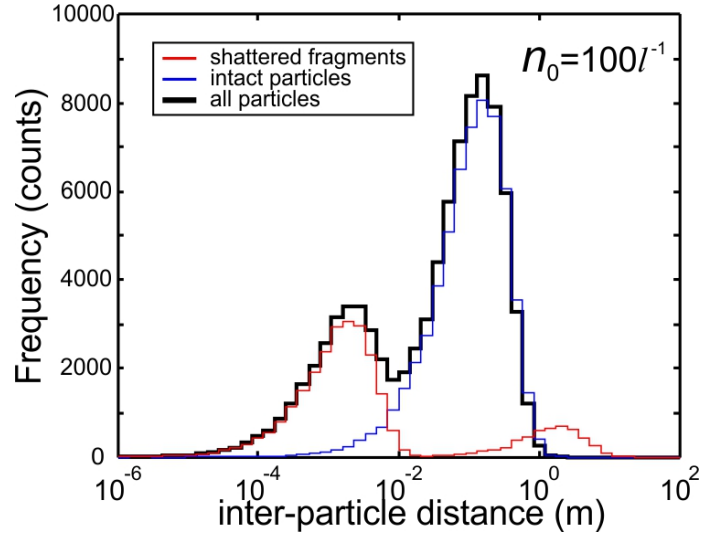

Figure 18. Results of Monte Carlo simulations of the distribution of inter-particle distance $\phi(\Delta x)$ for intact particles (blue), shattered particles (red), and all particles passed through the sample volume (black). The calculations were conducted for particle concentration $n_{0}=100 \mathrm{~L}^{-1}$

preted as a concentration of the particle's contribution in the effect of shattering, but not as a total concentration, which included small ice particles which do not affect shattering. For simplicity, the effect of the particle size distribution was not included in the simulation. It should be noted that the threshold $400 \mu \mathrm{m}$ refers to OAP-2DC. Probes with different pixel resolution, response time, and inlet configuration have different threshold sizes below which the effect of shattering becomes insignificant.

The analysis of the modeling results suggests that: (1) the number of counts in the long inter-particle distance mode is mainly controlled by the sample area $S_{0}$ and particle concentration $n_{0} ;(2)$ the number of counts in the short distance mode depends on the area $S_{\mathrm{sh}}$, which deflects particles towards the sample volume, the characteristic number of fragments per shattering event $\bar{N}_{\mathrm{sh}}$ and the particle concentration $n_{0}$. As demonstrated in Sect. $4, \bar{N}_{\text {sh }}$ is a function of particle size. It should also be noted that $\bar{N}_{\mathrm{sh}}$ depends on $S_{\mathrm{sh}}, S_{0}$ and the spatial proximity of $S_{\mathrm{sh}}$ to $S_{0}$. For example, as $S_{0}$ decreases the probability of passing at least two shattered fragments $\left(N_{\mathrm{sh}} \geq 2\right)$ through $S_{0}$ diminishes too. If $S_{0}$ becomes too small, most of the shattered artifacts will be associated with single particle shattered events $\left(N_{\mathrm{sh}}=1\right)$, that cannot be identified by the ITA. Therefore, it is anticipated that the inter-arrival time algorithm will be more efficient for OAP2DS with $S_{0} \approx 50 \mathrm{~mm}^{2}$, than for the FSSP with the sample area $S_{0} \approx 3 \mathrm{~mm}^{2}$. The number of shattered fragments viewed by the probe $N_{\mathrm{sh}}$ also depends on spatial separation of $S_{\mathrm{sh}}$ to $S_{0}$. Thus, if $S_{\mathrm{sh}}$ is located too far from $S_{0}$ then the shattered fragments may not have sufficient momentum to travel large distances across the airflow and reach $S_{0}$. Comparisons of the measurements of standard and modified probes demonstrated that $\bar{N}_{\text {sh }}$ strongly depends upon $S_{\text {sh. }}$. The reduced number of the shattered fragments $N_{\text {sh }}$ for the modified probe is ex- 

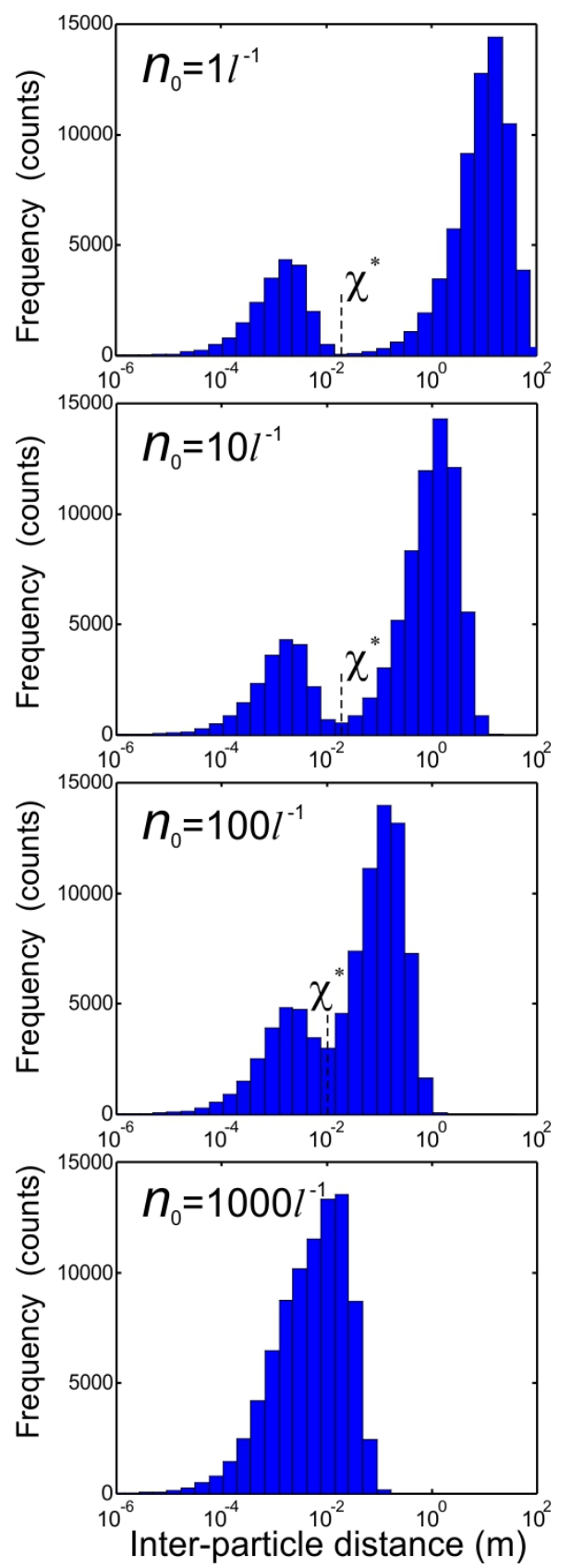

Figure 19. Results of Monte Carlo simulations of the distribution of inter-arrival time $\phi(\Delta x)$ for different particle concentrations (a) $1 \mathrm{~L}^{-1}$; (b) $10 \mathrm{~L}^{-1}$; (c) $100 \mathrm{~L}^{-1}$; (d) $1000 \mathrm{~L}^{-1}$.

plained by the fact that the antishattering tips have a significantly reduced shattering area compared to the standard tips.

\section{Conclusions}

Based on the analysis of data obtained with standard and modified OAP 2DC probes in a variety of ice cloud conditions and Monte Carlo simulations, the following conclusions have been obtained:

1. The inter-arrival time algorithm cannot segregate all shattering artifacts from the intact particles in principle. These limitations are imposed by the Poisson statistics of particle spatial distribution. It was demonstrated that the short inter-arrival times are not necessarily associated with shattering artifacts, and that shattered artifacts are not constrained to exhibit short inter-arrival times. In order to mitigate the effect of shattering, the interarrival time algorithm should be used together with other means, such as antishattering tips and special algorithms to improve its performance (e.g., reacceptance of intact particles, corrections for accepted singletons, etc.).

2. The inter-arrival time algorithm has a range of conditions when short and long inter-arrival modes are well separated and it can effectively segregate shattered artifacts and intact particles (e.g., low concentration).

3. The inter-arrival time algorithm has a number of limitations which under certain circumstances may significantly degrade its performance or even disable it. Such cases are relevant to the clouds with high particle concentration, when the spatial separation between shattered fragments $\Delta x_{\mathrm{s}}$ becomes comparable with the distance between intact particles $\Delta x_{\mathrm{i}}$, i.e., $\Delta x_{\mathrm{s}} \sim \Delta x_{\mathrm{i}}$. For mixed-phase clouds it may not be possible to use the inter-arrival time algorithm with probes that have a fine pixel resolution.

4. It was found that in clouds with particles with $D_{\max }<400 \mu \mathrm{m}$ the effect of shattering on measurements of the standard OAP-2DC can be neglected.

5. The inter-arrival distance and number of registered shattered artifacts is well represented by an exponential function. The slope of the distribution is a function of the characteristic particle size. The number of shattered fragments correlates with particle size.

6. The results on the statistics of shattering events open the door for a statistical simulation to study the effect of shattering on measurements. These studies could potentially be useful not only for developing correction algorithms for historical data sets but also for developing recommendations on how to design probes in the future to best accommodate the ITA.

This analysis of the efficiency of the inter-arrival time algorithm is based on OAP-2DC data. Nevertheless, most of 
the conclusions obtained in this study can be applied to other particle probes. In this regard it is worth mentioning that the effectiveness of the inter-arrival time algorithm depends on the pixel resolution, size of the sample area, the response time of the electronics and the inlet configuration.

Acknowledgements. The work of A. Korolev was supported by Environment Canada, Transport Canada and the US Federal Aviation Administration.

Edited by: A. Lambert

\section{References}

Baker, B., Mo, Q., Lawson, R. P., O'Connor, D., and Korolev, A.: The Effects of Precipitation on Cloud Droplet Measurement Devices, J. Atmos. Ocean. Tech., 7 1404-1409, 2009.

Baumgardner, L., Avallone, A., Bansemer, S., Borrmann, P., Brown, U., Bundke, P., Chuang, Y., Cziczo, D., Field, P., Gallagher, M., Gayet, J.-F., Heymsfield, A., Korolev, A., Krämer, M., McFarquhar, G., Mertes, S., Möhler, O., Lance, S., Lawson, P., Petters, M. D., Pratt, K., Roberts, G., Rogers, D., Stetzer, O., Stith, J., Strapp, W., Twohy, C., and Wendisch, M.: In Situ, Airborne Instrumentation: Addressing and Solving Measurement Problems in Ice Clouds, B. Am. Meteorol. Soc., 93, ES29-ES34, doi:10.1175/BAMS-D-11-00123.1, 2012.

Cooper, W. A.: Cloud physics investigation by the University of Wyoming in HIPLEX 1977, Bureau of Reclamation Rep, AS, 119, 321 pp., 1977.

Field, P. R., Wood, R., Brown, P. R. A., Kaye, P. H., Hirst, E., Greenaway, R., and Smith, J. A.: Ice particle inter-arrival times measured with a Fast FSSP, J. Atmos. Ocean. Tech., 20, 249-261, 2003.
Field, P. R., Heymsfield, A. J., and Bansemer, A.: Shattering and Particle Inter-arrival Times Measured by Optical Array Probes in Ice Clouds, J. Atmos. Ocean. Tech., 23, 1357-1370, 2006.

Jackson, R. C., McFarquhar, G. M., Stith, J., Beals, M., Shaw, R. A., Jensen, J., Fugal, J., and Korolev, A.: An assessment of the impact of antishattering tips and artifact removal techniques on cloud ice size distributions measured by the 2-D Cloud Probe, J. Atmos. Ocean., Tech., 31, 2567-2590, 2014.

Korolev, A. V.: Reconstruction of the Sizes of Spherical Particles from Their Shadow Images. Part I: Theoretical Considerations, J. Atmos. Ocean. Tech., 24, 376-389, 2007.

Korolev A. V., Strapp, J. W., and Isaac, G. A.: Evaluation of accuracy of PMS Optical Array Probes, J. Atmos. Ocean. Tech., 15, 708-720, 1998.

Korolev, A., Emery, E. F., Strapp, J. W., Cober, S. G., Isaac, G. A., Wasey, M., and Marcotte, D.: Small ice particles in tropospheric clouds: fact or artifact? Airborne Icing Instrumentation Evaluation Experiment, B. Am. Meteorol. Soc., 92, 967-973, 2011.

Korolev, A., Emery, E., and Creelman, K.: Modification and tests of particle probe tips to mitigate effects of ice shattering, J. Atmos. Ocean. Tech., 30, 690-708, 2013a.

Korolev, A. V., Emery, E. F., Strapp, J. W., Cober, S. G., and Isaac, G. A.: Quantification of the effects of shattering on airborne ice particle measurements, J. Atmos. Ocean. Tech., 30, 2527-2553, 2013b.

Lawson, R. P.: Effects of ice particles shattering on the 2D-S probe, Atmos. Meas. Tech., 4, 1361-1381, doi:10.5194/amt-4-13612011, 2011.

Strapp, J. W., Albers, F., Reuter, A., Korolev, A. V., Maixner, U., Rashke, E., and Vukovic, Z.: Laboratory Measurements of the Response of a PMS OAP-2DC, J. Atmos. Ocean. Tech., 18, 1150-1170, 2001.

Vidaurre, G. and Hallett, J.: Particle Impact and Breakup in Aircraft Measurement, J. Atmos. Ocean. Tech., 26, 972-983, 2009. 